\title{
Ever growing sensitivities
}

\section{Jaap Bregman}

ASTRON

Oude Hoogeveensedijk 4, 7991 PD Dwingeloo, The Netherlands

E-mail: bregman@astron.nl

\section{Bou Schipper}

ASTRON (retired)

Oude Hoogeveensedijk 4, 7991 PD Dwingeloo, The Netherlands

E-mail: b.a.p.schipper@gmail.com

\section{Wout Beerekamp}

ASTRON (retired)

Oude Hoogeveensedijk 4, 7991 PD Dwingeloo, The Netherlands

E-mail: whjbeerekamp@home.nl

\section{Arnold van Ardenne}

ASTRON

Oude Hoogeveensedijk 4, 7991 PD Dwingeloo, The Netherlands

E-mail: ardenne@astron.nl

\section{Teun Grit}

ASTRON

Oude Hoogeveensedijk 4, 7991 PD Dwingeloo, The Netherlands

E-mail: grit@astron.nl

\section{Marco de Vos}

ASTRON

Oude Hoogeveensedijk 4, 7991 PD Dwingeloo, The Netherlands

E-mail: devos@astron.nl

\section{Gie Han Tan}

Direct. Oper., ESO HQ, Karl-Schwarzschild Str. 2, 85748 Garching bei Munich, Germany

E-mail: ghtan@eso.org

\section{Nico Ebbendorf}

ASTRON

Oude Hoogeveensedijk 4, 7991 PD Dwingeloo, The Netherlands

E-mail: ebbendorf@astron.nl

\section{Jan-Pieter de Reijer}

ASTRON

Oude Hoogeveensedijk 4, 7991 PD Dwingeloo, The Netherlands

E-mail: reijer@astron.nl 


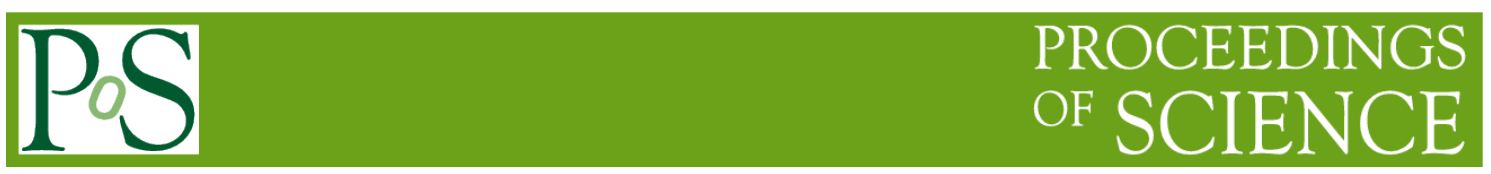

\section{Jürgen Morawietz}

ASTRON

Oude Hoogeveensedijk 4, 7991 PD Dwingeloo, The Netherlands E-mail: morawietz@astron.nl

\section{Albert Jan Boonstra}

ASTRON

Oude Hoogeveensedijk 4, 7991 PD Dwingeloo, The Netherlands

E-mail: boonstra@astron.nl

\section{Johan Pragt}

ASTRON

Oude Hoogeveensedijk 4, 7991 PD Dwingeloo, The Netherlands

E-mail:pragt@astron.nl

\section{Jan Idserda}

ASTRON (retired)

Oude Hoogeveensedijk 4, 7991 PD Dwingeloo, The Netherlands

\section{Eddy Elswijk}

ASTRON

Oude Hoogeveensedijk 4, 7991 PD Dwingeloo, The Netherlands

E-mail: elswijk@astron.nl

\section{André Gunst}

ASTRON

Oude Hoogeveensedijk 4, 7991 PD Dwingeloo, The Netherlands

E-mail: gunst@astron.nl

50 Years Westerbork Radio Observatory, A Continuing Journey to Discoveries and Innovations Richard Strom, Arnold van Ardenne, Steve Torchinsky (eds) 


\section{Ever growing sensitivities}

\section{Chapter 10.1 Ever growing sensitivities}

Jaap Bregman*

$\mathrm{I}$ nstallation of the multi-beam Apertif system on the Synthesis Radio Telescope in Westerbork (WSRT) completes the full range of efficient image forming capabilities realized by inventive implementation of available technology. It is therefore appropriate to look back on 50 years of development of the receiving systems that followed the construction of ten fixed radio telescopes and a few more on a rail track, completed in 1968 three years after the tender.

We will follow the development of the sensitivity of the complete interferometric receiver system as stepwise upgrading of its constituent parts using the then most advanced and affordable technology. As with LOFAR, it is interesting to note that ASTRON developed the base technology of the latest WSRT receiving system by its technology development program funded in 1996 to prepare for next generation radio telescopes. For WSRT, the concept of a Phased Array in the focal plane of each reflector creates many adjacent beams on the sky. It transformed our synthesis telescope into one where 37 synthesized instruments based on advanced signal processing techniques are now at work simultaneously.

\section{Introduction}

A synthesis radio telescope like the WSRT makes, just as other telescopes, pictures of the sky. This is realized by combining the signals from a number of reflector antennas, and processing these combinations with a computer system that finally produces the sky pictures. The underlying combination principle for electromagnetic radiation comprising the received wave front was first analysed in 1934 by Pieter van Cittert, a physicist from Utrecht University in The Netherlands. The Groningen University Professor and Nobel Laureate Frits Zernike simplified the derivation in 1938 now known as the Van Cittert-Zernike theorem.

* ASTRON, The It took until 1962 when Professor Martin Ryle at Cambridge University demonNetherlands strated this principle for the first time with just two radio telescopes, which 
brought him a shared Nobel Prize. The WSRT, operational in 1970, had 12 telescopes on a perfectly straight East-West line of 1,6 km length. With its correlation receiver system for the $21 \mathrm{~cm}$ band, it was the first 'picture machine' available to the scientific community internationally. As a result, some of the most gifted young radio scientists settled down at the Universities in Leiden and Groningen contributing to the world fame of Dutch radio astronomy.

It was especially the sensitivity of the WSRT, an order of magnitude greater than of existing university instruments, which formed the basis of international success. The collecting area of its reflector together with the equivalent system noise of its receiver in the focus is the first component which determines the sensitivity of a synthesis instrument. Secondly, the number of telescope combinations, the bandwidth and the duration of an observation determine the noise in a synthesis image. More instantaneous beams just speed up the observing of large fractions of the sky. Data processing provides the third component in the sensitivity by removing the many artefacts in a synthesis image that increase the effective noise level above the theoretical one. This latter aspect is discussed in Chapter 6

\section{Global development path of the WSRT}

The invention of the transistor in 1947 started a new era for designing electronic circuits. At first for professional and military equipment, but in 1956 the first transistor radios appeared on the consumer market. The demand for semiconductor components in consumer products was an important stimulus for mass production providing by 1963 high frequency transistors for TV applications. These devices were not only cheaper than the electronic amplifier tubes, but also much smaller and produced far less heat allowing the design of compact units needed for our interferometric receiver system. Only the low-noise amplification of the extremely weak antenna signals from the sky at frequencies above $1 \mathrm{GHz}$ required a different concept i.e. the parametric amplifier with a special semiconductor diode.

This new and affordable technology allowed us to start in 1965 with design and building the first large synthesis radio telescope in the world. It is not surprising that The Netherlands could make this daring step, since the natural gas reserve in Groningen just started to deliver, and was also the largest reserve in the world at that time.

Five instrumental development periods can be identified as determined by technological possibilities on the one hand and by Astronomical research requirements and their budgets on the other hand.
Instrumental development and enabling technologies:

1965 - '70 Discrete transistors for amplification and correlation

Homemade quasi-degenerate parametric low-noise amplifiers

1970 - '76 First chips in digital lag-correlator operating at 1- and 2-bit digitization

Commercial non-degenerate parametric low-noise amplifiers

1976 - '82 Custom chips in digital complex-correlator

Cryogenic cooled parametric amplifiers and low-noise FETs below $1,8 \mathrm{GHz}$

1986 - '98 Full custom chips for digital lag-correlator Cryogenic cooled low-noise FETs

2005 - '15 8-bit Fast Fourier Transform correlator in Field Programmable Gate Arrays

Ultra-low-noise HEMTs at L-band in Focal Plane Array

WSRT Heydays

We may consider a first phase from 1965 until ' 82 during which the upcoming semi-conductor technology is quickly implemented to take the lead in the radio aperture synthesis branch in astronomical research and kept it for a long time. An important driver was the American plan to build a synthesis array with 27 telescopes with 3 arms, each $21 \mathrm{~km}$ long. This Very Large Array, the VLA, would achieve more than twice the sensitivity and 25 times sharper view due to its longer baselines. The first realization activities for the VLA started in 1973 and the first phase of operation began in ' 80 .

However, the WSRT was in 1976 still far ahead with just two additional telescopes on the rail track and a digital lag-correlator providing 2560 spectral channels for twice the number of unique baselines. In 1979, two movable telescopes were relocated to an additional rail track $1.3 \mathrm{~km}$ to the East extending the length of the synthesis array to about $2.8 \mathrm{~km}$. This would provide sufficient resolution at $21 \mathrm{~cm}$ to see details in nearby galaxies that would only just pop-up in line emission even with the most sensitive cryogenic cooled receivers of the future. Therefore, the WSRT would stay competitive in all areas as the VLA would be hit by the same brightness sensitivity limitation.

Much more important was the increase in sensitivity of the receivers in the focus of the telescopes. That sensitivity has two components of which the noise of the first amplification stage is the most important one. The effective noise temperature was a factor of two reduced by replacing the quasi degenerate parametric amplifiers by nondegenerate ones. Further improvement resulted from cryogenic cooling of the low-noise amplifier stages in the four movable telescopes. 


\section{Multiplying in Cotton}

Around 1977 a retrofit of the workhorse $6 \mathrm{~cm}$ Westerbork receivers was planned in order to introduce the latest technologies and improve the performance. In these receivers, an auxiliary signal is generated such that the resulting lower frequency carries the astronomical signals through long cables to the central building for further processing. The modules that generate this auxiliary signal were called multipliers because the output frequency was n times the input frequ ntimes the input frequency fed to the receiver. These modules were not commercially available largely because of their specific and critical character, including a requirement for very high stability. As a consequence, they were designed and built in the lab.

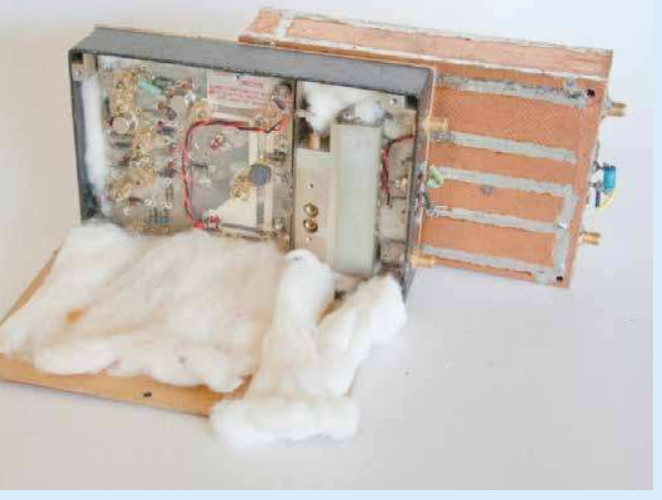

However, and a bit to our surprise it turned out that by now there was an opportunity to buy them based on our custom specifications. The UK company Bradley was selected and tasked to provide the $14 \mathrm{X}$ frequency multipliers with precise specifications with respect to frequency tuning, temperature, and phase stability. Phase stability is especially important for an interferometer like the WSRT! It tur out pretty soon that with regard to this stability requirement, the company had hardly any experience. In order to minimize the delays of the ongoing project with a clear demand for quick availability to the (staff) astronomers, I was asked to visit the company I vas asked to visit the company every 2 weeks to monitor progress and advise them on the design. This, of course, fit our experience very well. In the end and in spite of my input, it turned out that the company was unable to achieve the required temperature-and phase stability After some intense nerotiations with the com pany, Jean Casse, then head of the pany, Jean Casse, then ho labs succeeded to purchase them for half the price, leaving me with the issues to resolve.

With some creativity using industrial cotton to fully stuff the modules so as to stabilize the temperature and hence the phase, it ended up working propery! After also sop the unwanted radiation from the modules with help of Harm-Jan Stiepel and the use of neatly soldered copperfoil around the modules, this adventure page could be turned. The picture tells the story working properly! After also solving
The second important factor is the effective frequency bandwidth, which is in practice limited by the digital correlator. There, the total bandwidth is divided into a large number of sufficiently small channels to properly image the velocity distribution in line emission over objects. This signature of source motion arises due to the Doppler effect, requiring a large number of frequency channels at the expense of processing power.

Although the $10 \mathrm{MHz}$ bandwidth of the digital lag-correlator was sufficient for line studies and an improvement over the $4.2 \mathrm{MHz}$ of the 1970 correlator, it would by no means be competitive with the planned $80 \mathrm{MHz}$ of the American VLA. Therefore, it was decided to build a separate digital correlator providing that same bandwidth, which became operational in 1983 .

Consolidation

After a break in the receiver development for the WSRT and the first successes of the VLA, it became clear that all telescopes needed cryogenic cooled receivers and that many more spectral channels were required for significant scientific results. In addition, the periodic change of the three sets of receivers could not support the wish for rapid change in frequency for objects that changed their wide-band spectral behaviour. Therefore, plans started in 1986 to construct a so-called Multi Frequency Front End (MFFE) where 5 feed systems could be quickly rotated to the focus of the reflector supporting 9 different frequency bands. With the MFFE, the sensitivity would only be a factor 1.6 worse after 12-hour observing compared with an 8-hour synthesis at the VLA at the most crucial $21 \mathrm{~cm}$ band. However, combining additional 12-hour observations with different positions of the movable telescopes provides a regular set of baselines. This greatly reduces the artefacts in a wide field synthesis image, which has always been the hallmark of the WSRT.

This flexible MFFE together with a first segment of the new lag correlator became operational in 1999. Only after completion of the new signal conversion system in 2001 the full number of 262,144 spectral channels became available for 8 bands of $20 \mathrm{MHz}$ reaching over 1 million channels when 8 times $5 \mathrm{MHz}$ was selected. These two workhorses, together with refurbished telescope reflectors, new drives and a new on-line control system, operated smoothly until the shutdown in 2015 to prepare for the next step.

From multi-frequency to multi-beam

After a second break in receiver and correlator development for the WSRT, it became time for a last upgrade where the WSRT could remain in serious competition with the upgraded VLA after 2010 and until the planned Square Kilometre Array takes over after 2020. In 2005 the focused design and development started of a revolutionary multi-beam telescope system using ASTRON's 
base technology for phased array antennas and Fourier transform correlators. After earlier engineering demonstration tests, the system implementation on the telescopes started in 2015 combined with a major telescope maintenance and improvement cycle. This Apertif system will commence full operations in 2018. Instead of one, Apertif creates 37 synthesis arrays, where each field has $350 \mathrm{MHz}$ bandwidth and 10 million spectral channels for its 91 baselines. Its purpose is to image the neutral hydrogen in all galaxies to an unprecedented depth in the sky, as well as the time varying universe of which full scientific accounts are presented in Chapter 15 .

\section{Sensitivity Progress over Time}

We summarize the increase in performance in a sensitivity for continuum surveying "Pcs" given by the square root of the product of number of interferometers N, total bandwidth B and number of beams per telescope "Nb", and then divided by the system noise temperature Ts at $21 \mathrm{~cm}$. The last column gives the impressive relative increase "RI" with a factor of over 500x since 1970 (first line) of the WSRT system. The increase in processing power is even more impressive (see below).

\begin{tabular}{lllllccl}
\hline Year & Ts & N & B & Nb & Pcs & RI & remarks \\
1970 & 260 & 20 & 4.2 & 1 & .0353 & 1.0 & quasi-degenerate paramps \\
1974 & 85 & 20 & 4.2 & 1 & .0515 & 1.5 & non-degenerate paramps \\
1977 & 85 & 40 & 10 & 1 & .235 & 6.7 & 4 movables and DLB \\
1980 & 50 & 40 & 10 & 1 & .4 & 11.3 & cryogenic movables \\
1983 & 50 & 40 & 80 & 1 & 1.13 & 32 & DCB \\
1998 & 27 & 91 & 160 & 1 & 4.47 & 127 & MFFE and DZB using all baselines \\
2015 & 50 & 66 & 350 & 37 & 18.5 & 524 & Apertif uncooled HEMT \\
\hline
\end{tabular}

The first $21 \mathrm{~cm}$ receiver and correlator system for the WSRT took 4 years to develop prototypes and to build a complete system for 12 telescopes. It had one beam on the sky, a system noise temperature of $260 \mathrm{~K}$ using only 20 baselines with 4 polarization channels and 4.2 $\mathrm{MHz}$ bandwidth.

Fifty years later the Apertif receiver and correlation system used again 12 telescopes but it took some 13 years from early design to operation. It provides about 3.3 times as many interferometers for each of its 37 beams on the sky. The receivers are 5 times more sensitive and the correlator provides for each interferometer about 115,000 spectral channels in an 83 times wider band. This is more than 370 million correlations compared to only 80 in 1970 and reflects a doubling every 2.2 years, close to the prediction by Moore's law for digital processing performance.

Although the inflation corrected costs for equipment and labour are roughly equal, the time from conception to completion differs by a factor three. The rea- son for this delay is that critical specialists had to share time with other projects as well, while the impact of all the new observing and calibration aspects on the control system was underestimated.

\section{The first steps in Synthesis imaging}

The call for tender in April 1965 to construct 12 telescopes with $25 \mathrm{~m}$ diameter reflectors and erect them at a site near Westerbork, followed shortly after completion in 1964 of the one-mile synthesis radio telescope at Cambridge University, UK. The one-mile array had three telescopes with $18 \mathrm{~m}$ diameter reflectors, of which the middle one movable on a rail track of $800 \mathrm{~m}$ starting near one telescope along an almost East-West line. Seen from the polar star at a large distance every telescope describes in 12 hours Earth rotation, half a circle around the other telescopes. In this way, the linear distances between the telescopes fill the 2-dimensional plane of the correlation function with half circles. The two correlations between the movable and the two fixed telescopes produce two baseline tracks for each setting of the moveable telescope. After 60 steps along the rail-track a complete 2-dimensional correlation function with 120 halfcircular tracks on a regular concentric grid is obtained. According to the Van Cittert-Zernike theorem, a Fourier transformation would give a projected image of the full sky but with enhanced intensity in a field defined by the beam profile of the telescopes that tracked a specific sky location. Also, every object in the field is broadened by the much narrower beam profile of a telescope with a diameter of one mile.

We do not only get a nice image with all the objects in the field, but each object is surrounded by a set of so called concentric grating rings. When the step along the rail track equals the diameter of the telescope, the rings of an object in the centre fall just at the edge of the field defined by the telescope beam. For smaller increments, the rings widen up, for larger increments, the rings narrow in and could disturb the object of interest.

The synthesis array in Westerbork would get 10 telescopes at a distance of $144 \mathrm{~m}$ from each other and 2 movable ones on a rail-track of $300 \mathrm{~m}$ all within a few $\mathrm{mm}$ from a perfect East-West line. With 20 unique correlations between the fixed and movable telescopes, 6 steps of $12 \mathrm{~m}$ on the rail would also provide a regular concentric grid with 120 half-circles. Twice the collecting area per telescope and the same receiver technology then provides double sensitivity and makes the telescope a factor 10 faster for a full synthesis. In practice, increments of 18 or $36 \mathrm{~m}$ were chosen with lower sensitivity and additional disturbing grating responses in the produced images, but many more fields could be observed in a given period.

This speed was important, since the English had already completed their ${ }_{5} \mathrm{C}$ catalogue at $408 \mathrm{MHz}$ before 1970 and had just begun with $21 \mathrm{~cm}$ observing. A new window on the sky opened up and which ones of the 100,000 visible fields 
on the sky would contain groundbreaking scientific discoveries waiting there for eons to show their secrets?

\section{Continuum system 1970}

An interferometric receiver system has different parts. The signals from the radio source enters the Front-End (FE) in the prime focus box of each telescope and while keeping its information moves to the Back-End (BE) in the central building. There the telescope signals are brought together with coaxial cables for further processing. In the FE the sensitive low-noise pre-amplifier stage and further amplification stages overcome the transport and other losses.

The most critical element in a FE is the low noise preamplifier. Reduction of the noise by factor two has the same effect on the sensitivity as increasing the collecting area by a factor two. This would be very costly, and would also reduce the size of an observed field by a factor two.
In 1966 a team at Dwingeloo Radio observatory led by Prof. Muller developed a parametric amplifier. This state-of-the-art device had a bandwidth of about $50 \mathrm{MHz}$ around $1.410 \mathrm{MHz}$ and an equivalent noise temperature of $55 \mathrm{~K}$. This amplifier was of the quasi-degenerate type, which meant the output also contained an amplified version of a folding input band. The consequence for interferometric and spectral line work is that all noise contributions of sky, antenna filters and connectors ahead of the paramp input and of the paramp itself have to be added and then doubled resulting in an effective system noise temperature of $260 \mathrm{~K}$. However, this was sufficient for the moment since a nondegenerate type could replace these amplifiers in a later phase.

The Back-End had different parts to amplify the signals and shape them to 4.2 $\mathrm{MHz}$ bandwidth, crossmultiply and integrate them to interferometer correlations, all in analogue transistor technology. A scanner system read the correlated signals with a digital voltmeter under control of a Philips $\mathrm{P}_{92} \mathrm{O} 2$ computer that wrote the data to magnetic tape.

\section{Fixing the Mesh:}

\section{"Het Boutjesproject"}

After 8 years operation of the WSRT, it turned out that the reflecting mesh glued to the individual panels was coming loose in rapid progression. Thus was born "The Fixing Project" ("Het Boutjesproject" in Dutch) placed under the capable technical leadership of Ton Wolferst' (then WSRT lead mechanical engineer) and Wout Beerekamp (then leading WSRT operations).

The solution was to fix the stainless steel mesh frames to the $2 \mathrm{~mm}$ thick panels using small selftapping screws. These were to be mounted by

compressed air driven handheld driling tools. In an incredible display of

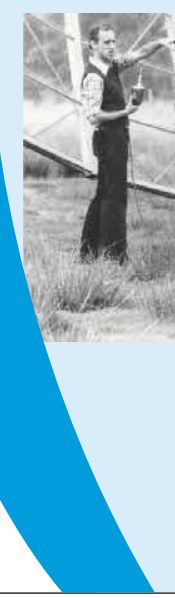
Simkin as hard working volunteer drilling screws at ground level, and (right) high up, Cees Slottje and Hans van Someren Greve. In one incident, a basket was acciCente minimal (and there were no injuries to our volunteers).

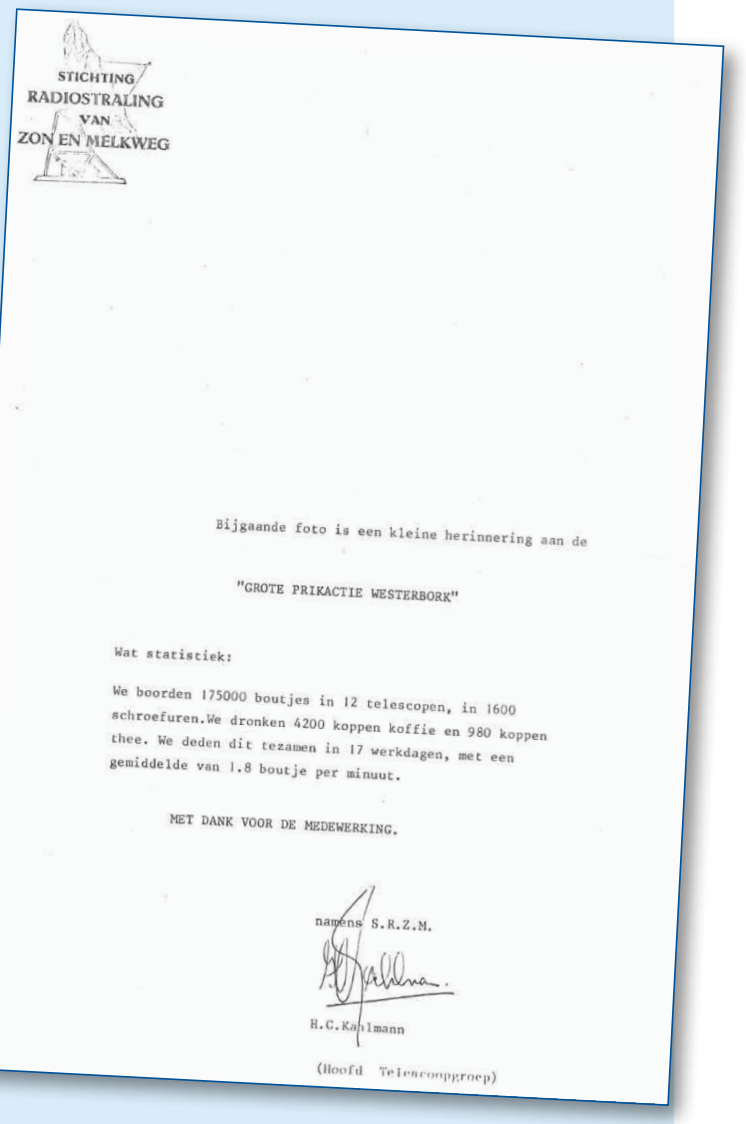

communal solidarity, many astronomers, students and engineers from all around the national astronomica community answered our distress call for help to restore "their" monumental WSRT. Volunteers Were housed in caravans and tents on the nearby camping sites.

Besides using our own cherrypicker, three others were hired to get the job done in minimum time. One of those was capable to stretch to $25 \mathrm{~m}$ height to reach the outermost panels ensuring a great view, but there was some difficulty to convince our volunteers to get into the pickers basket! However, after a short and intense course by Ton and Wout, most volunteers accepted the challenge to do the job way up there, moving the cherrypickers to all heights while others remained on solid ground.

In the end Hans Kahlmann, head of the Telescope Group, expressed his appreciation by a written declaration to all who brought the WSRT back on track (see facsimile). Our volunteers drilled 175,000 screws, in 1600 hours over 17 days. And by the way, they also 
With 12 telescopes 66 cross-correlations can be formed. Since the 10 fixed and 2 movable telescopes have regular distances, 46 combinations appear in every observation. Therefore, only 20 combinations are unique and different for shifted movable settings that densify the regular grid of correlation tracks. This notion considerably simplified and reduced the correlation system.

Every FE had two orthogonal dipole antennas and required four correlation channels per interferometer. Since a correlation signal has two components, 160 channels had to be read every 10 seconds.
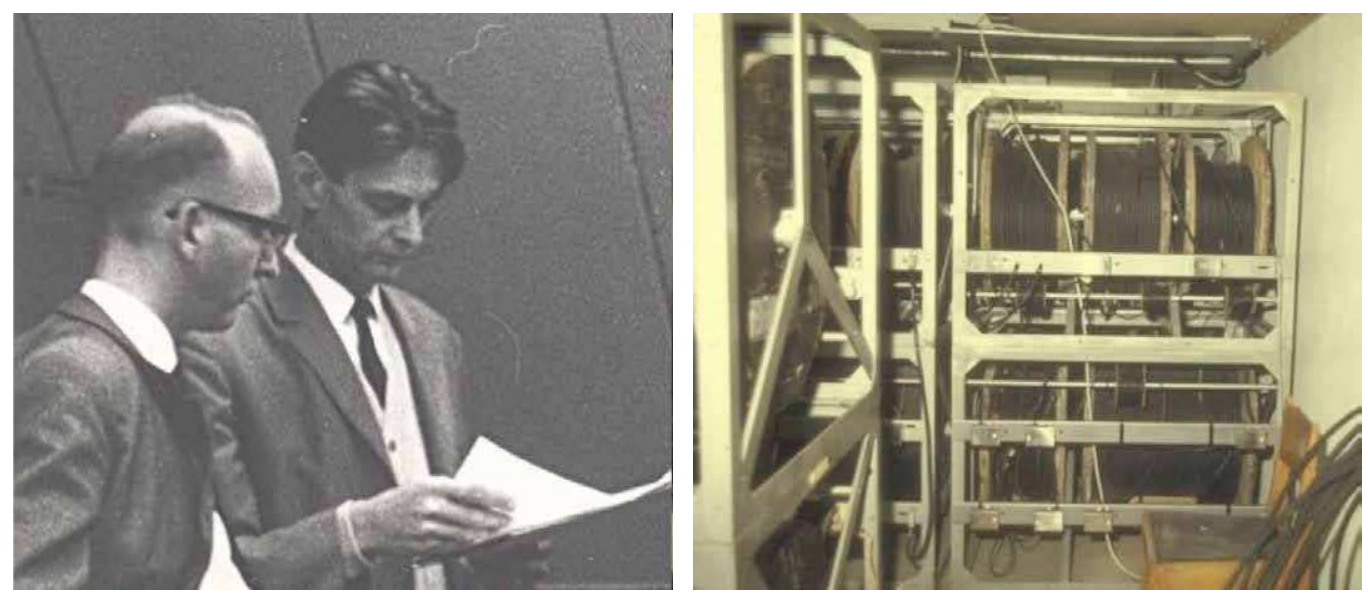

Figure 1 (left): Prof C.A. Muller left and receiver engineer A.C. Hin right

Figure 2 (right): Delay cable rack with spools for 640 $320,320,160,80$, 40,20 and $10 \mathrm{~m}$ $\max 1590$ the correlation system by signals from the control computer, which depend on
The delay racks are an important and bulky subsystem of the BE. During an observation, the telescopes follow a track on the sky, which means that during the rising part the eastern telescopes receive a wave front earlier than the western ones. This needs compensation for proper correlation of all spectral components in the $4.2 \mathrm{MHz}$ wide signal, which happens in steps of $10 \mathrm{~m}$. The gradual path change between steps causes phase changes that are corrected in the instantaneous position of the observed field on the sky.

\section{Line system 197}

The first surprise in 1970 was the discovery of a galaxy where the spiral arms in continuum radio emission did not coincide with the bright arms illuminated by starlight. It was therefore essential to observe line emission that would reveal location and velocity of the gas in the arms.

The rest frequency of emission by neutral Hydrogen is at $1420.406 \mathrm{MHz}$, but appears at higher frequencies for approaching velocities due to the Doppler shift. The typical range of frequencies in spiral arms of a galaxy is of order $250 \mathrm{~km} / \mathrm{s}$ and at least 16 frequency channels are required to record a spectrum including some empty channels for a proper reference.
The correlator system provided for $2 \times 10$ interferometers with four polarization channels each and could be reconfigured into $2 \times 5$ interferometers with 8 spectral channels of $129 \mathrm{kHz}$ wide and $190 \mathrm{kHz}$ separation for a single polarization. This corresponds to a channel width of $27 \mathrm{~km} / \mathrm{s}$ and velocity steps of $40 \mathrm{~km} / \mathrm{s}$ spanning a range of $280 \mathrm{~km} / \mathrm{s}$. For a complete spectrum, two observations are required shifted by $95 \mathrm{kHz}$ to fill the gaps between the filters. Compared to the continuum bandwidth of 4.2 MHz with full polarization on all telescopes, a factor 11 in sensitivity is lost for a single line channel. This loss needs compensation by additional observations providing more baselines and by decrease of the system noise temperature.

Purchasing two non-degenerate parametric amplifiers with $42 \mathrm{~K}$ noise temperature permitted the modification of one polarization in each Front-End of the two moveable telescopes, bringing their system noise temperature down to $75 \mathrm{~K}$. When combined with 5 fixed telescopes at $260 \mathrm{~K}$ it translated in a geometrical mean system temperature of $140 \mathrm{~K}$. Line observations started by the end of 1971.

These amplifiers were extremely expensive. After inflation correction this amounts to a quarter million Euro, to be compared with only a few Euros for the very low noise transistors for Apertif in 2015. Still, it was only $0.3 \%$ of the total cost of the WSRT while almost doubling its performance. The funds therefore had to be found as the Astronomical community needed a sound explanation for the spiral structure of gas and stars in galaxies, a challenging situation that had to be addressed!

\section{$6 / 50 \mathrm{~cm}$ system}

The first switchable receiver system became operational in 1972 and allowed comparing the intensity of sky sources at three different frequencies which is important for the identification of the radiation mechanisms. The field at $50 \mathrm{~cm}$ wavelength was a factor 2.3 larger than at $21 \mathrm{~cm}$ allowing larger objects to be imaged. At $6 \mathrm{~cm}$ the resolution was a factor 3.5 higher showing more imaging detail, with the $21 \mathrm{~cm}$ receiver in between. Figure 3 shows the $6 / 50 \mathrm{~cm}$ receiver suite in the Westerbork Observatory building.

\section{$6 \mathrm{~cm}$ subsystem}

The commercial quasi-degenerate double stage parametric amplifiers had a limited bandwidth around 4,995 $\mathrm{MHz}$ and realized an effective system noise temperature of $220 \mathrm{~K}$. In preparation for the new digital line Back-End the bandwidth of the complete receiver chain was $10 \mathrm{MHz}$.

An upgrade program in 1977 installed new non-degenerate parametric amplifiers realizing a system noise temperature of $120 \mathrm{~K}$ and $100 \mathrm{MHz}$ output bandwidth. An additional modification in 1980 to the multiplier ensured stable 


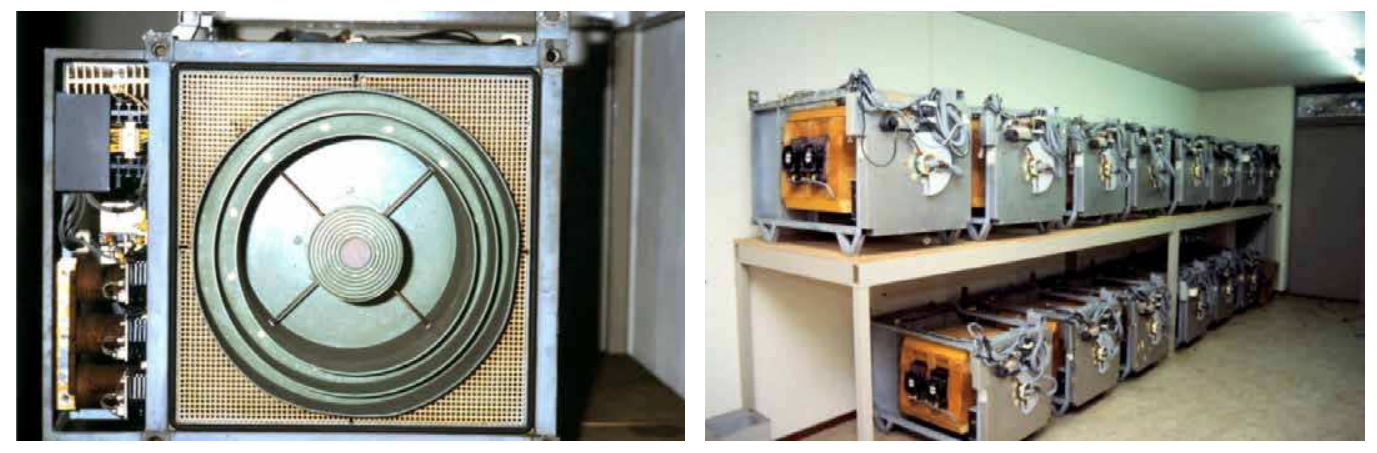

Figure 3 (left): Looking into the $6 / 50 \mathrm{~cm}$ FrontEnd (right): Rack with $156 / 50 \mathrm{~cm} F \mathrm{FE}^{\mathrm{s}}$ in Westerbork

working over the full tuning range from 4,770 to $5,020 \mathrm{MHz}$, making two recombination lines accessible.

$50 \mathrm{~cm}$ subsystem

The $50 \mathrm{~cm}$ receiver used a bipolar low noise transistor, which in combination with the narrow band input filter realized a system noise temperature of 350 K. This high loss filter centred at $610 \mathrm{MHz}$ had to pass the $5.5 \mathrm{MHz}$ wide TV channel 38 , left free for radio astronomy, and reject other TV channels. Unfortunately a TV transmitter at about $150 \mathrm{~km}$ distance suffered from internal interference causing spurious signals in our channel 38 , which was cured by an additional high power filter at the transmitter. The new digital line BE provided in 1977 a $2.5 \mathrm{MHz}$ filter allowing much steeper filtering in addition to the FE filter eliminating weak interference from two German TV transmitters. A further upgrade in 1981 used a Ga-As FET as low-noise input transistor with a far larger signal dynamic range, allowing placement of the narrow band TV filter after the low noise transistor. The consequence was a much improved system noise temperature from $350 \mathrm{~K}$ to $110 \mathrm{~K}$.

\section{$21 \mathrm{~cm}$ upgrades}

A first step in 1971 replaced in two FEs the quasi-degenerate parametric amplifier of one polarization by a non-degenerate one from Micromega having $42 \mathrm{~K}$ noise temperature and a frequency range from $1.365 \mathrm{MHz}$ to $1.425 \mathrm{MHz}$. Another important step was that the single dipole was no longer rotatable and had a straight low-loss connection to the paramp, which resulted into a system temperature of $\sim 75 \mathrm{~K}$.

A second step was made in 1974 when all $21 \mathrm{~cm}$ FEs got non-degenerate paramps. However, the limited rotation over 45 degrees caused higher losses and resulted in $85 \mathrm{~K}$ system noise temperature.

At the last step was in 1984 when the complete electronics, except for the paramps, was replaced to increase the output bandwidth to $100 \mathrm{MHz}$ in preparation for the new wide band continuum backend.

\section{Mr. Correlator}

It is my great pleasure to contribute this page in honour of Albert Bos, ASTRON's own Mr. Correlator. Of course Albert is retired now and following many other aspirations and activities, but when he allows himself to look back, he may smile at his technical legacy of many state of the art digital lag correlators which he and his team built in the which he and his team built in the
ASTRON lab. One can even say ASTRON lab. One can even say
that the digital age started at ASTRON with Albert's first correlator in 1976. It was equipped with the so called "Bos" chip which was the very first in-house designed TTL chip, and it remained available on the commercial market for some years. This first Digital Line Backend (DLB) operated for a decade into the mid-eighties, with the help of some improvements along the way. Albert acquired his

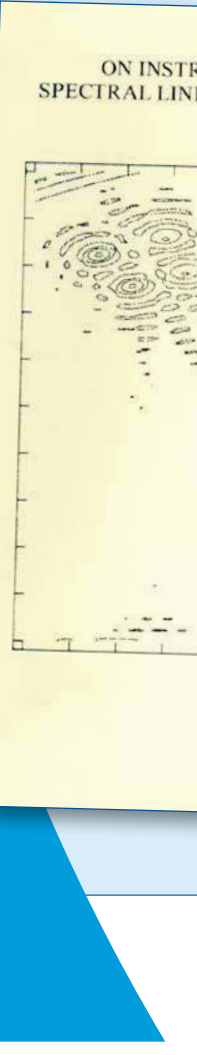

PhD from Leiden University in 1982 based largely on his success with the DLB. The thesis was focused on the instrumental effects on line synthesis observations as a result of the many imperfections in the observing system, including correla. tion and processing. Albert's work on the DLB was recognized with the 1996. The DLB was followed by the DXB whe DIB was followed by the DXB with a sidetrack to make the correlator (the "DAS") for the JCMT, the submm Hawaiian telescope for which, rather unknown by many. ASTRON built the complete

$350 \mathrm{GHz}$ receiver-IF-correlator system in the mideighties. As the making of correlators took quite some time partly because of the flexibility resulting from the large number of functional configurations, his last correlator was called the DZB. This top of the line system was equipped with a custom specific chip based on the Bos architecture in collaboration with JIVE and others including Haystack/MIT in the US. It was a formidable achievement, which together with frontend upgrades, concluded the WSRT system upgrade around 2000 . Albert was extremely dedicated and often stayed late in the Observatory building working on system improvements. Such was his enthusiasm and ded upon opening one of the correlators 19 " racks Albert would jump out! Time passes on and we can truly say that the ultra-stable W/SRT performance from which many astronomers have benefited owe a great thanks to Albert's correlators! prestigious national Veder awad in ication that sometimes I felt that 


\section{Digital Line Back-end and Recirculation}

The Digital Line Back-End or DLB was a major step forward to handle 10 fixed and 4 movable telescopes at a maximum bandwidth of $10 \mathrm{MHz}$. It improved the continuum sensitivity only by a factor 1.4 due to additional digitizing losses at the highest bandwidth. The system has 320 blocks of 8 complex spectral channels that allow various distributions of the 2,560 channels over baselines and channels. One extreme has even 5120 channels in autocorrelation mode on a single telescope, and another configuration could cross correlate all telescopes together. The latter configuration was instrumental in developing the so-called redundancy calibration technique. Since the fixed telescopes are placed on a regular grid some baselines appear multiple times. Since the sky response is only baseline dependent, comparing the various results allows interpreting actual differences as disturbances from telescope and atmosphere (see Chapter 6).

The design and development of a prototype started in 1970 and the complete system became operational in 1977. There are 4 racks each with 4 crates containing 20 correlation cards each as can be seen in Figure 5. About 10,000 wires connect the boards in the crates and have a crossed layout as seen in Figure 6 to prevent crosstalk. To prevent the radiation of unwanted interference cause by the digital equipment they are placed in a closed metal cabin. See Figure 4 also showing the in-between boards wiring technique called wire-wrapping. This kept digital engineers Arie Doorduin, Hans Weggemans, Roelof Kiers and perhaps others, quite busy at the time!
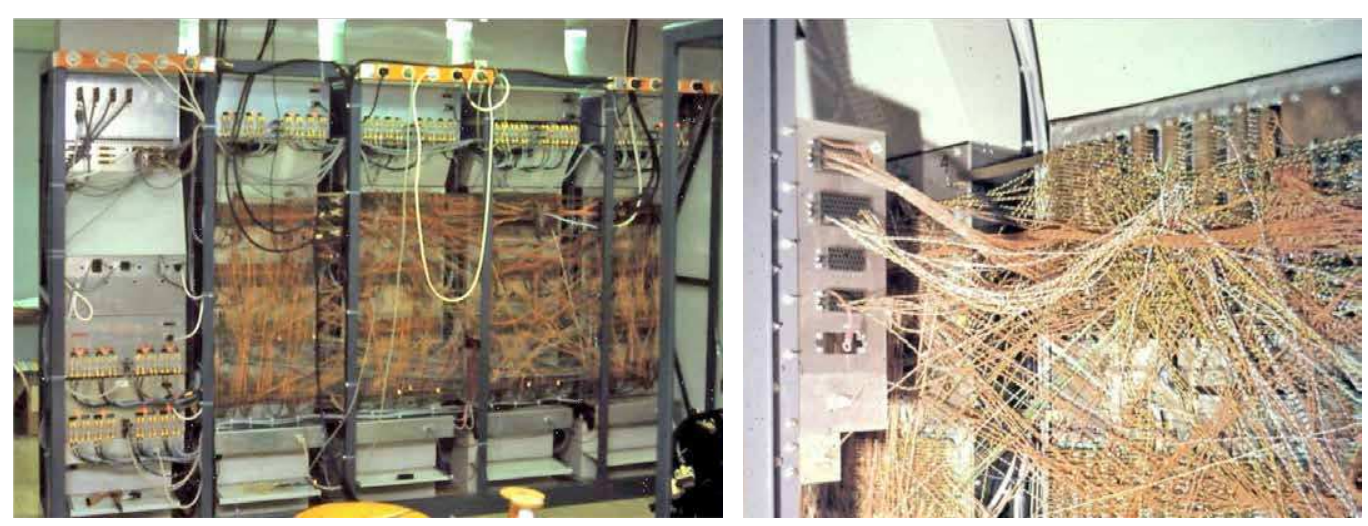

The Digital Line Back-End has four subsystems. Following the signal chain the first is where the Intermediate Frequency (IF) signal from each telescope is converted to a video signal of sufficient strength. Also, the bandwidth is limited from a selectable range from $10 \mathrm{MHz}$ down to $78 \mathrm{kHz}$ in steps of a factor two. The second subsystem contains the digitizers where each video signal is digitized to 2 bit, a sign bit and a high/low bit. High means higher than the root mean square value of the noise signal. The third subsystem is the digital delay and the fourth system is the correlator, where the signals from different signals paths are cross-multiplied and integrated.

Multiplying 1 and 2 bit signals is realized with a few logic gates while integration requires a counter. In 1970, these functions were already available in Schottky TTL chips. The digital lag correlator has a shift register where 16 steps of a signal stream are stored and clocked through. The 16 delayed samples are all cross-multiplied with the same sample of the second data stream and at each lag integrated in a separate counter. The samples are delayed in steps of $50 \mathrm{~ns}$ as defined by the $20 \mathrm{MHz}$ clock, which is according to the Shannon theorem sufficient for sampling of a noise signal of $10 \mathrm{MHz}$ bandwidth. The WienerKhinchin theorem, also from 1934 when Van Cittert gave his first formulation for wave fronts, states that Fourier transformation of 16 samples of an auto-correlation function gives 16 spectral channels of the input signal. Cross-correlation results in 2 numbers for only 8 spectral channels reflecting the complex nature of a signal with intensity and a phase.

\section{Recirculation option}

Correlating two polarizations for the fixed movable combinations provided in full 2-bit mode only 16 spectral channels, which is marginal in many applications. Since most line work is done with $2.5 \mathrm{MHz}$ total bandwidth, sampling at $10 \mathrm{MHz}$ is, according to the Shannon theorem, overdone. In fact, processing power of the correlator is wasted by repeating unnecessary operations. With an additional memory in the input data stream, only every fourth sample of the 2.5 $\mathrm{MHz}$ stream is stored. Then, processing a block with a four times longer time sequence at full speed provides a four times longer spectrum in a larger output buffer. In this way, the number of processed lags doubles for every halving of the input bandwidth. Finally, a maximum recirculation factor 16 was chosen providing 40,960 spectral channels in 1-bit mode. Team Albert Bos dubbed the additional unit DXB. It became operational in 1985, marking maturity of the lag principle.

It is interesting to note that the world's first multipurpose single board PC based on the Motorola 6800 microprocessor using an on-board OS9 real time operating system, might have been developed by Rob Millenaar in 1979. It was used in the delay controller system of both DLB and DCB (see below).

\section{Cryogenic $6 / 21 \mathrm{~cm}$ with $18 \mathrm{~cm}$ option on the movable telescopes}

In the late sixties, it was already clear that the system noise temperature was dominated by receiver losses that could only be reduced by reducing the actual temperature. This started the development of cryogenic cooling in 1970 (see Chapter 3). Lead by Bert Woestenburg, this resulted in an operational system in 1979 with $6 / 21 \mathrm{~cm}$ cooled receivers on the four movable telescopes. 


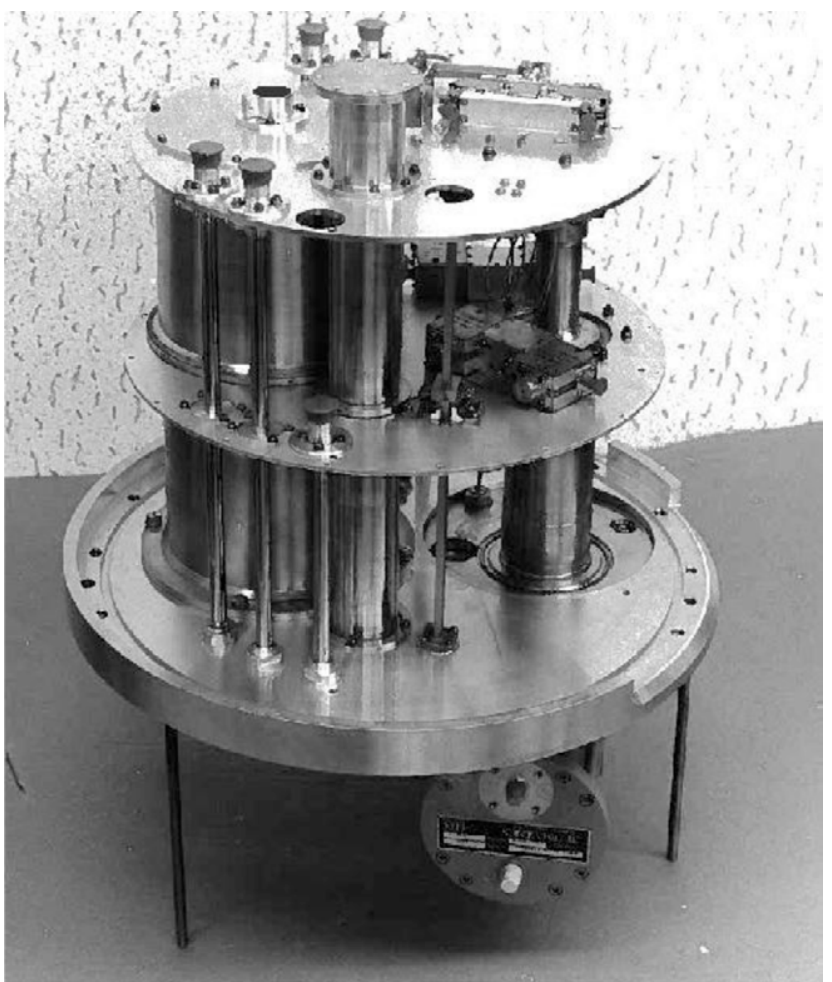

Figure 5 shows the CTI cooling system with two plateaus at the right. The top plateau at a temperature of $20 \mathrm{~K}$ has two non-degenerated AIL parametric amplifiers for $6 \mathrm{~cm}$ operation. The second amplification stage using Ga-As FETs is located on the second plateau at $70 \mathrm{~K}$. The complete chain provides on the telescope a system temperature of about $55 \mathrm{~K}$. Together with the $120 \mathrm{~K}$ system on the fixed telescopes the geometric mean of $81 \mathrm{~K}$, is a factor 2.7 better than in 1972 .

After lots of tough lab work, including the removal of unwanted resonances only noticeable with closed cryostat cover, the $21 \mathrm{~cm}$ up-convertor provided a receiver noise temperature of $13 \mathrm{~K}$ and $29 \mathrm{~K}$ system temperature on the telescope, a new milestone in 1980 Westerbork. Together with the fixed telescopes, the equivalent was now $50 \mathrm{~K}$ system, a factor 4.6 improvement over 1970.

$18 \mathrm{~cm}$ option

For VLBI observation with the WSRT in tied array mode an $18 \mathrm{~cm}$ observation capability was important. The $18 \mathrm{~cm}$ band was added by replacing the $21 \mathrm{~cm}$ up-convertor for a self-developed wide-band Ga-As FET amplifier. This $18 \mathrm{~cm}$ system needed a different dipole system mounted outside the cryostat. The uncooled connection cables raised the system temperature to $55 \mathrm{~K}$.

\section{Digital Continuüm Backend}

By 1973 our American colleagues embarked on a project for a serious synthesis radio telescope. This Very Large Array or VLA, would have 27 telescopes with $25 \mathrm{~m}$ diameter reflectors and a suite of receivers in the Cassegrain focus, becoming operational after 1980 . With cooled receivers on all telescopes and making all possible correlation between them, the system would provide after an 8 hour synthesis a factor 1.7 improvement in sensitivity over the WSRT with a $4 \mathrm{X} 12$ hour synthesis at the $21 \mathrm{~cm}$ line. For continuum observing a bandwidth of $80 \mathrm{MHz}$ was planned, giving an additional improvement factor of 2.8. To stay competitive for continuum observing it was therefore essential to increase the $10 \mathrm{MHz}$ bandwidth of the DLB. This was done by a new, additional BackEnd, the DCB. In 1983, after 7 years of design, prototyping, and mass production, the Digital Continuum Back-End, the DCB, started regular operation at the WSRT. Figure 6 shows the happy DCB team.

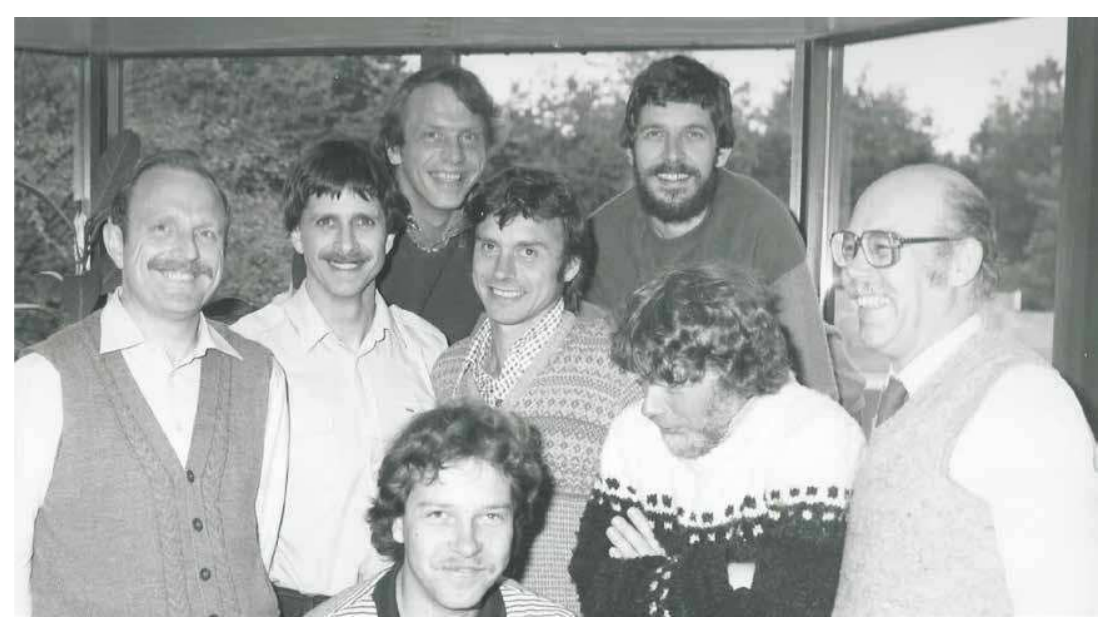

The Low Power Schottky TTL chips still had the same maximum clock speed of $20 \mathrm{MHz}$ as used in the DLB. This required division of the $80 \mathrm{MHz}$ band in sections of $10 \mathrm{MHz}$, each with their own set of digitizers, delay modules and cross correlators. Since the $80 \mathrm{MHz}$ range in the $21 \mathrm{~cm}$ contained parts with strong radar signals, each band had filters to select $10 \mathrm{MHz}$ or $5 \mathrm{MHz}$ bandwidth operation at a dedicated centre frequency.

The 1970 system correlated a set of fixed telescopes against a set of movable telescopes. To obtain the two components of the correlation function, the signal of a movable telescope was duplicated, but with an additional phase shift of 90 degrees. A comparable trick was required when every telescope needed correlation against all other telescopes. The trick was found by using the principle of a modified Weaver mixing scheme, where the first step was done in the analogue domain at conversion of the IF signal to video, and the second step 
in the digital domain after correlation. The Weaver down conversion process uses two mixers that each fold the $10 \mathrm{MHz}$ wide signal band around a selectable IF frequency providing two $5 \mathrm{MHz}$ video bands of which one with a godegree phase shift. This approach requires digitization of twice as many signals, but still using the $20 \mathrm{MHz}$ clock results in oversampling. Fortunately, twofold oversampled 3-level correlation has $8 \%$ digitization loss comparable to 4 -level (2-bit) cross-correlation. This 3-level correlation gave considerable simplification of the digital correlator circuitry, but doubled the number of video convertor, digitizers, and delay blocks. Correlation was now not between two $10 \mathrm{MHz}$ signals but between two pairs of $5 \mathrm{MHz}$ signals requiring four cross-correlators instead of one.

Despite the integrated chips, the cables remained a nightmare as indicated by Figure 8.
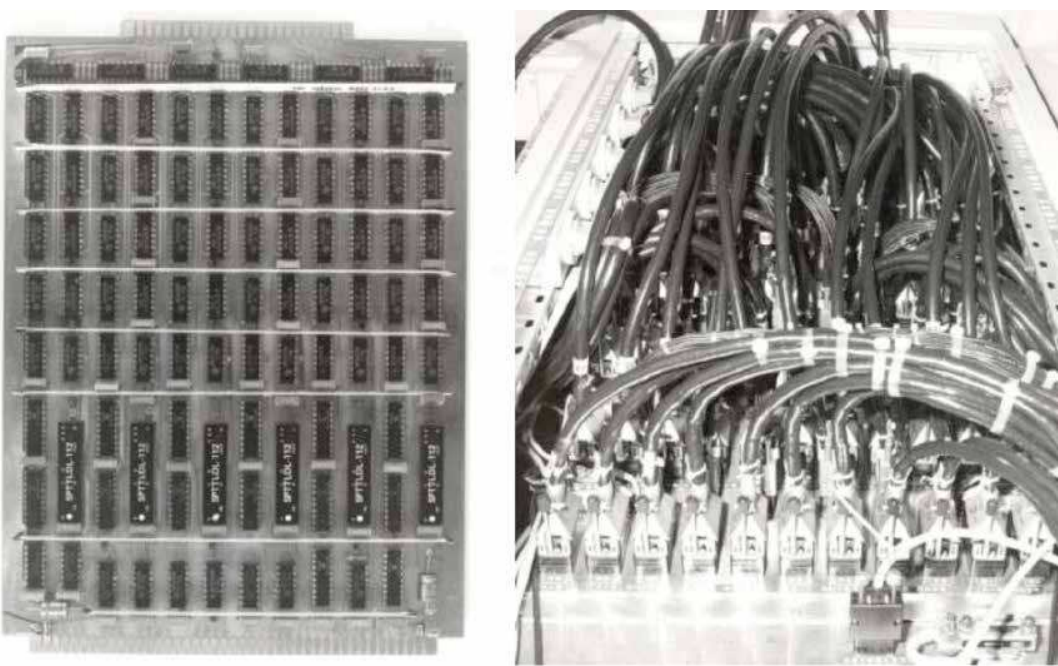

\section{Controlling the WSRT}

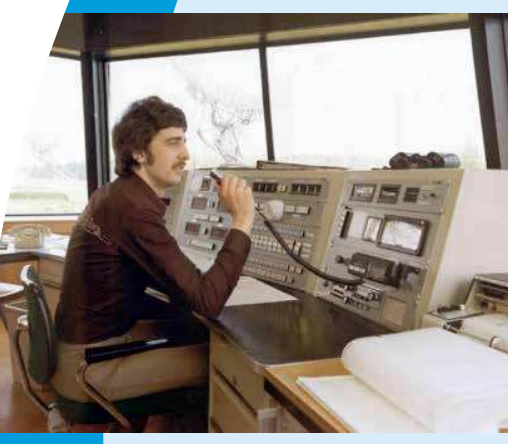

into one long punch paper tape. On to be read in before you could start Friday, the 16 bit computer with 32 testing your modifications. This kByte memory read this paper tape, happened with a fast optical reader started the observations and the re- and afterwards the tape ended up in sults were stored on magnetic tape. a large basket. "So you want to learn These tapes were sent to Leiden on how to program? Then help me by Monday for further analysis.

Programming was also time consuming. All software was written in assembler (machine instructions one by one) and was typed on punc cards. Then the stack of punch cards with source code was fed into the computer for compilation. The result was a punch tape with one or more routines. W/ith every error the more roulines. With every error, the was very time-consuming, but the instrument was in production and we were pressed for time. When you finished all the compilation work, the small paper tapes had to be assembled into one "sector" punch tape of about $10 \mathrm{~cm}$ diameter. In the end, 32 of those big punch tapes had

es had in that period and I owe him a lot.

verted and combine
Teun Grit

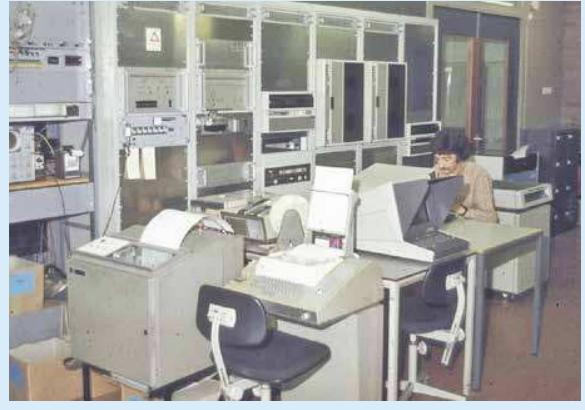
winding those punched tapes in that basket." As a junior, you did not dare to refuse!

Meanwhile, "new" HP1000 com puters were installed and they wer equipped with a Fortran compiler. left the assembler language for what it was and focused on Fortran $4 X$. Programming was fun and therefore I started an "AMB|" study with the languages Alool and Fortran. officially started as a programmer in 1981. For about 25 years, Hans 25 years, Hans van Sormeren Greve and I shared the same room and while Hans worked on the on-line (control) software, I focused on the off-line (processing) software. Hans was my superviso
When the mathematics became problematic, Hans would supply me with a well-tested routine. On the other hand, Hans was not happy with system administration and so we formed a good team. In those years, I also managed the RTE and HP-UX systems. In the mid-1990s, the first Suse Linux systems arrived.

One of those HP1000 systems has lasted for 25 years as an embedded system in the Digital Continuum Backend. We did not dare to turn it off anymore because we were afraid that the power supply would fail! Such a HP1000 computer had a fixed and a removable hard drive of
2 MByte. I remember the crash of my first hard drive around 1980. While I was working, I suddenly heard a screeching sound coming out of the disc. Fortunately, there was a backup on tape. I still have the crashed disk at home

The internet started in 1983. Some years later, the WSRT was connected We could send 75 bytes / sec and receive 1200 bytes / sec. This made ASTRON one of the first internet users in the Netherlands. As a result, my passport photo was for ears the most popular passport photo of the Netherlands. Every time one searched for "pasfoto", my until years later.

When the HPgooo arrived around 1990, languages such as C, Perl and
Pascal were also used. With the ascal were also used. With the arrival of the Telescope Managemen guage became popular. The Fortran programs from the 1980 a use as the IWWOS (the WWSRT CO
Software) package. I am proud of at. That is legacy code of almost 40 years!

In 2007 I started working full-time as a system administrator for LOFAR and WSRT. Today the WSRT systems such as ARTS have $128 \mathrm{CB}$ of memory, which is more than 4 million times more per machine than the Honeywell/Philips 9200 computer from 1970. Soon we will , , $000,000,000$ bytes) than my first work drive!

When retiring in May 2020 I will leave ASTRON with pain in the heart. ASTRON was and still is, a fantastic institute and I feel very lucky to be part of it!

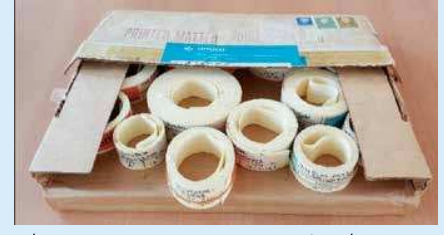

The 1976 paper tapes contain plot tines that are still in use in IWOS. 
The resulting four numbers needed proper recombination to produce the two numbers representing the complex correlation of a single $10 \mathrm{MHz}$ band. This operation was in essence a complex multiplication and required another one to perform the fringe de-rotation before final integration in computer memory. A Hewlett Packard 21MX computer, integrated in the correlator system, got a dedicated high-speed operating system to perform these operations every $0.2 \mathrm{~s}$, before finally transferring the integrated data every $10 \mathrm{~s}$ to the on-line control processor.

Another novelty was the custom-specified ULA chip from Ferranti. This Uncommitted Logic Array contained sufficient gates to perform high-speed integration for a set of eight three-level correlations. This allowed much cheaper further integration by a low-speed counter section that provided sufficient precision for the $0.2 \mathrm{~s}$ read-out cycle.

Each of the eight band sections had 1024 cross-correlators for 256 interferometers of which 160 for the fixed-moveable combinations in full polarization. The remaining channels covered most of the dual polarization combinations between the redundant baselines in the fixed and the moveable telescope subsets.

\section{$90 \mathrm{~cm}$ receiver system}

Initial plans for a $90 \mathrm{~cm}$ system took a few years of cooperation with the Technical University of Eindhoven to develop a compact feed system with dipoles in the launcher part on the FE frame and a ring on the focus box. The challenge of obtaining proper electromagnetic operation without impairing observing with the other FE series successfully concluded in 1983 with a full series of $92 \mathrm{~cm}$ Front-Ends.

Although the FET pre-amplifiers had a noise temperature of about $100 \mathrm{~K}$, the narrow-band antenna filter of $3 \mathrm{MHz}$ at the low frequency deuterium line at $327 \mathrm{MHz}$ raised the system temperature on the telescope to about $200 \mathrm{~K}$. The measurement of the deuterium (a heavier version of hydrogen) and hydrogen ratio is of importance to cosmology.

The WENSS program, a large Survey of the Northern sky at $50 \mathrm{~cm}$ and $90 \mathrm{~cm}$, required a lower noise temperature at $92 \mathrm{~cm}$ comparable to $110 \mathrm{~K}$ at $50 \mathrm{~cm}$. An uncooled FET amplifier in 1988 already showed a low noise temperature of $25 \mathrm{~K}$. Together with a new lower loss $5 \mathrm{MHz}$ input filter the system noise temperature was $135 \mathrm{~K}$ measured on the telescope.

The last upgrade in 1993 provided a FE bandwidth up to $80 \mathrm{MHz}$ relevant to support dispersion correction for a pulsar detection program. It turned out that the $86 \mathrm{~cm}$ band between $310 \mathrm{MHz}$ and $390 \mathrm{MHz}$ could be used very well by careful setting of the eight $5 \mathrm{MHz}$ DCB bands, although it is an assigned aircraft communication band. In addition, the fluctuations in total power of the whole band posed no problems to the IF system, which allowed removal of the high-loss narrow-band filters in the FE. Together with the most recent FET's the system noise temperature on the telescopes was $100 \mathrm{~K}$

\section{Multi Frequency Front End}

Changing frequency band required replacing the FE in the focus for all 14 telescopes, an operation taking a few days once every few months. However, for many scientific programs it is essential to compare images at different frequencies made at about the same time. This requires a single frontend to be capable of changing frequency. The plans for such a front end that would handle 9 frequency bands ranging from 0.3 to $8.3 \mathrm{GHz}$, and the highest ones with cryogenic cooled preamplifiers, started in 1988. As a full account of the MFFE is given below, only a few milestones are given here.

The prototype cryogenic MFFE demonstrated a system temperature of $27 \mathrm{~K}$ at $21 \mathrm{~cm}$ in 1994 . Around 2000 all 14 telescopes had a complete MFFE covering 9 frequency bands. Five bands have cryogenic launchers and amplifiers at $21 \mathrm{~cm} / 18 \mathrm{~cm} / 13 \mathrm{~cm} / 6 \mathrm{~cm} / 3.6 \mathrm{~cm}$, and four noncooled amplifiers at $92 \mathrm{~cm}$, $49 \mathrm{~cm}$, UHFlow and UHFhigh.

In June 2015 the MFFE era came to an end to start preparing for the multibeam receivers. Two MFFE's (plus some spares) remain in operation for VLB and e.g. Galileo Satellite observations, on telescopes "o" and " 1 ".

\section{DZB, the last lag correlator}

The first ideas for the DZB correlator date back to 1992 when cooperation with MIT/Haystack started to develop a full custom integrated circuit containing 512 cross-correlation cells. This CMOS chip would run at a maximum clock speed of $40 \mathrm{MHz}$ to support a signal bandwidth up to $20 \mathrm{MHz}$. The chip was based on the famous Bos architecture developed at ASTRON in 1986 for a16channel chip running at a maximum clock speed of $55 \mathrm{MHz}$. This chip got worldwide usage and was used on the vertical board (Figure 12 left), which shows the typical configuration of boards on a backplane for use in a crate.

The new chip included additional features developed by MIT/Haystack for VLBI applications. Just as for the previous chip, there was worldwide interes and five different Astronomical institutes used the correlator boards each containing 32 chips. The WSRT and the Smithonian Submillimeter Array at Mauna Kea used direct telescope input, while JIVE, Haystack Observatory, US Naval Observatory, and MPfRA at Bonn used the signals from MKIV tape recorders for VLBI processing. See Figure 9 (right picture). 


\section{Developments in programming; Encapsulating legacy sotftware}

It must have been early 1995 that I sat in the office of Arnold van Ardenne discussing the WSRT upgrade when at some point conclud ed that something seemed to be missing. Almost all hardware systems were being either replaced and at the very least seriously revamped. Of course those changes had software repercuss dentified, and each of them could be implemented individually. That was the way things had always been done. Software follows hardware: new modes are added to the exis ing programs, new fields are allocated in the real-time memory. This had worked in the past most people were convin now as well. We had our doubts.

What seemed to be missing was a systems approach. The software running the WSRT had grown organically over more than a decade. There was no guarantee, either formal or heuristic, that it would continue to do so. Thus yet anothe subproject was added to the grand WSRT upgrade program: the Telescope Manager System, TMS. The big idea was to do things different, to professionalize the way we did software development. In retrospect, there were indeed several various new elements. Whether the shook the way we did software de velopment to their foundations, I'll leave to the reader. But they certainly where new in 1995, and they are useful to reflect on even now.

First was the choice for an object oriented design approach. This is not the same as "using $\mathrm{C}++$. " The TMS team made a serious effort to follow the formal methods that had been laid out by Rumbaugh in 1991. I still think this was a major improvement, mainly because it forced us to think in terms of architectures and interactions before we started coding. In 1997, when TMS development was well underway, we did some analysis on rework on infrastructural modules. It turned out that the amount of rework in modules with fully worked out designs was about $10 \%$ (one of every ten lines of non-comment code modified after initial release). The amount of rework in modules with marginal designs was up to 100\% (almost every line of code modified!)

Another element of change was the use of external consultants. They were of course received with great suspicion. To start with, they did not understand a thing about radio astronomy, so what good could they do (we of course knew a great deal of software engineering.....). Soon several colleagues realised this weakness provided an opportunity as well, in that these consultants, being good listeners, were quite willing to be educated. This in tum ed other colleg led ocher collegues into some deep reflections on the economic models of the firms employing the consultants. Apparently unaware of all this turmoil they were creating, the external experts came up with several object oriented architectures that formed the basis of the Telescope Manager System. In my recollec- tion, the most important thing they introduced was the use of multiple perspectives on a complex system. We've brought that to the next leve when applying IEEE standard 1471 100 for the Architectural Design of Software Intensive Systems to LOFAR.

In addition to bringing in experts from outside, we also brought in software packages (other than operating systems and compilers). The core of the "old" WSRT software was a large shared memory block that contained all the vital parameters. This was first mapped on an object oriented model of the WSRT and then implemented in a commercial real-time database. This

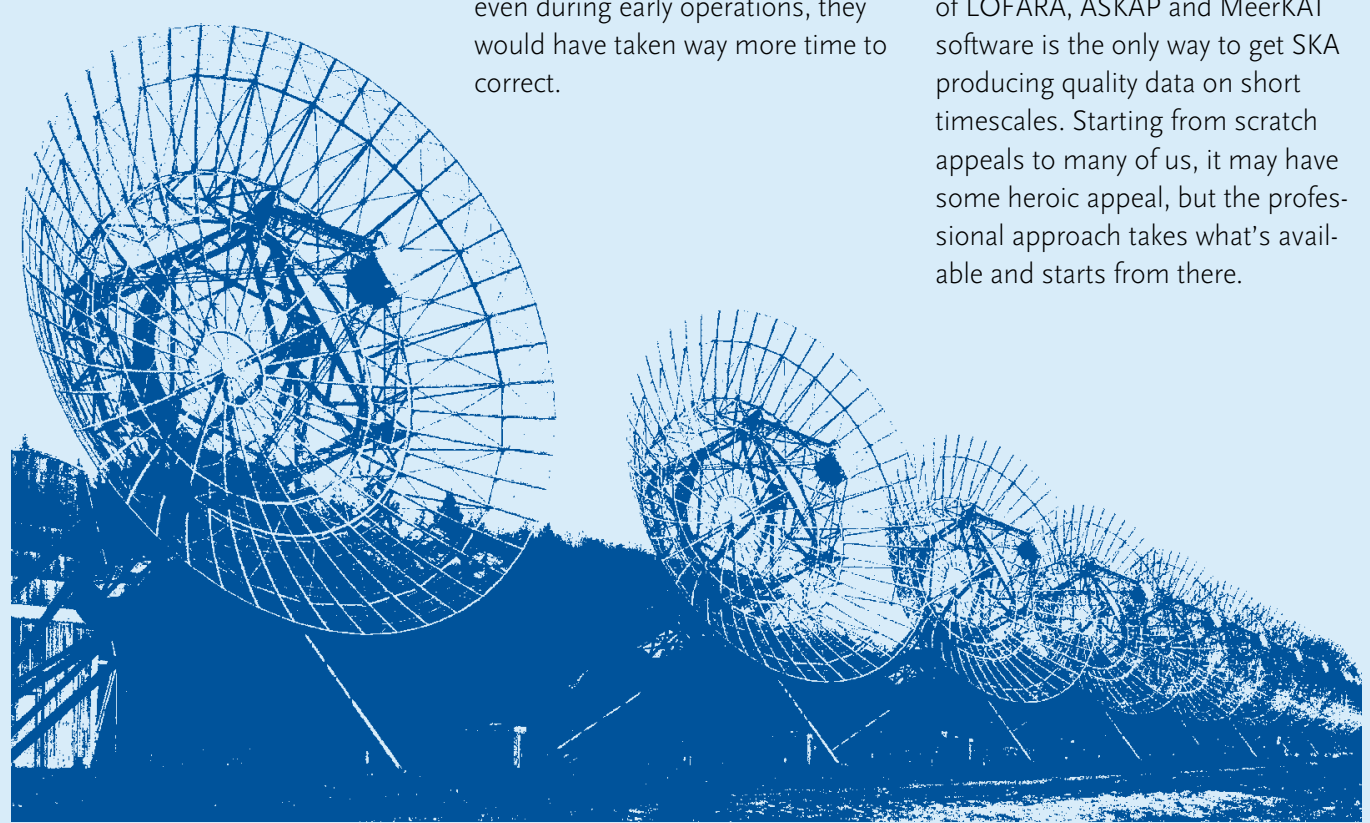

was a major change with a very high impact. The database came with a lot of nice features, like graphical dashboards, browsers and triggers. But for those who were used to a single big table, the added flexibility was hardly gain. However, this major change prepared us for was essential to handle the much more complex LOFAR system.

A final element of change was the introduction of peer reviews and change control. This may sound trivial now, but I can still hear people complain about colleagues looking at "their" code. With inspections in TMS we found on average two errors per non-trivial function before actual test. If these errors before actual test. If these errors even during early operations, they would have taken way more time

The TMS development enabled many new operating modes that would at best have been very difficult to implement in the old architecture. They showed us the strengths and weaknesses of forma methods and of external experts and tools. But they also showed us how important it is to maximise continuity when possible. One of the key design decision we made in the TMS project was to wrap wherever possible the existing software. Maximizing reuse by encapsulating legacy software is to be seriously considered for any major software intensive project. It helps has a high coh form of libraries like CASA Core), but it should be considered anyway. Im convinced that proper reuse only way to get SKA$$
\text { Tasonas }
$$
the distributed control model that 

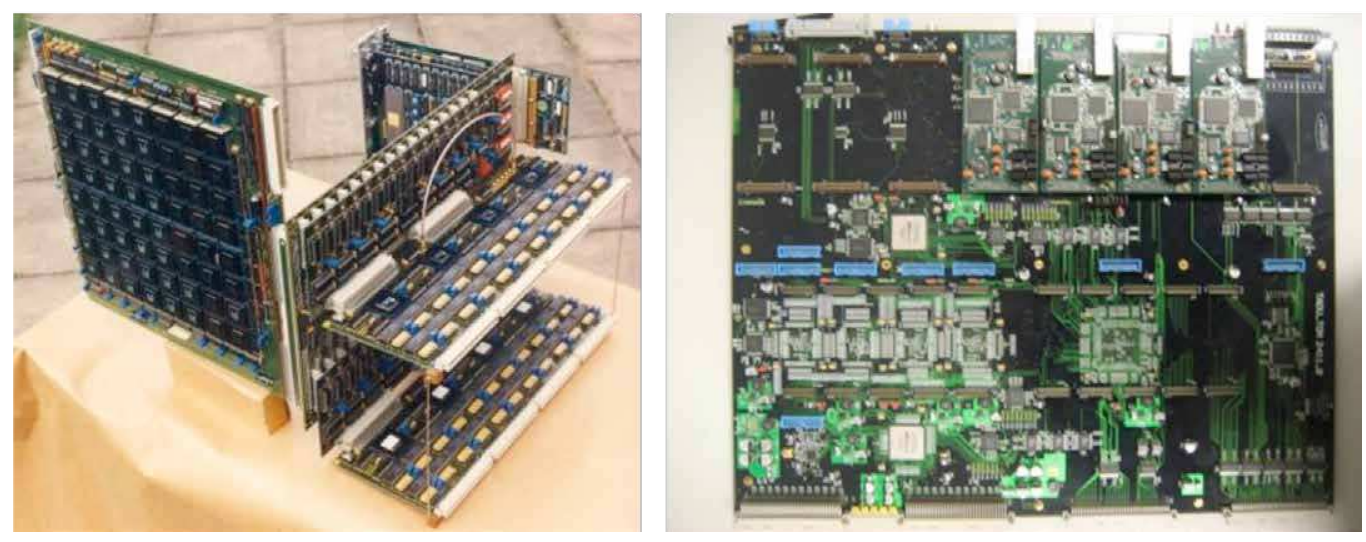

\section{Ever growing sensitivities}

\section{Chapter 10.2 The Multi Frequency FrontEnds; The ultimate single pixel Receivers for the WSRT}

Gie Han Tan*

Figureg: (left) Correlator board (the "DAS") build for the
James C. Maxwell Telescope on Haw in the eighties and (right) a DZB board again with custom

The WSRT would get a system with 1024 correlator chips and additional memory to support a maximum recirculation factor 16 . The new system had to replace DCB and DXB, make all correlations between 16 dual polarization inputs, including the auto-correlations, and provide for $160 \mathrm{MHz}$ bandwidth of the MFFE signals as well as for higher spectral resolution. The first segment of the DZB became operational in 1998 using one section of the DCB broadened to a bandwidth of $20 \mathrm{MHz}$. The system was completed in 2001 with a video converter system for 16 dual polarized telescopes signals. Each signal could be separated into 8 bands of $20 \mathrm{MHz}$ bandwidth down selectable to $156 \mathrm{kHz}$ in steps of a factor two.

The insert on page 175 shows ASTRON's Mr. Correlator, Albert Bos, who was responsible for all lag correlators.

From multi-frequency FE to multi-beam system the next stage

In 2006 plans started to succeed the MFFE's and the DZB with a system that would give the WSRT a competitive edge over the upgraded VLA (now the JVLA) expected to be operational by 2010 . This is Apertif using results from the ASTRON technology development for the SKA wide band aperture arrays with Vivaldi antennas (see Chapter 15). tarting at the end of the eighties and continuing during the nineties of the previous century the Westerbork Synthesis Radio Telescope (WSRT) underwent a major upgrade. The purpose was to overhaul it thoroughly and, more importantly, to put it again at the forefront of radio astronomy.

The ASTRON Strategic Plan issued in 1986 and updated in 1988, identified the need for this major upgrade and the ASTRON Board approved the plan for the design and construction of the ultimate single pixel receivers for the WSRT: The Multi Frequency Frontends (MFFE).

The major effort of this general system upgrade was the development and construction of new frontends for all 14 telescopes. The MFFEs would provide hugely increased frequency coverage. The frequency agility of the new frontends was one of the main advantages enabling new areas of astronomical research while at the same time eliminating the need for multiple sets of receivers. Apart from the huge cost saving and operational advantage, the MFFE construction with a single cryostat while extremely complicated, proved to be reliable and sturdy.

\section{Science drivers}

The astronomical community played the key role in defining the requirements for the MFFE. The obvious one was to improve the sensitivity in the existing WSRT receiver bands by applying the latest technological developments, e.g. High Electron Mobility Transistors (HEMT), using cryogenic cooling in all frontends Thus far only the receivers of the four movable telescopes were cooled.

A major challenge was to increase the frequency coverage of the frontends to satisfy evolving and new interests from the astronomers. At the high frequency end, beyond the existing $6 \mathrm{~cm}$ band, the need for a $3.6 \mathrm{~cm}$ receiver band covered interests in observing spectral lines and VLBI observations. The decision 
to the ground radiation. While the very low system temperatures on the Very Large Array (VLA) with full metal panel reflectors could simply not be reached, the substantial WSRT collecting area $\left(14 \times 490 \mathrm{~m}^{2}\right)$, nevertheless allowed a very reasonable sensitivity.

Primarily driven by the interest to observe red-shifted neutral hydrogen, there was a strong desire to expand the frequency coverage at the low end. The WSRT had a long and successful history in observing the $\mathrm{HI}$ line with the various $21 \mathrm{~cm}$ frontends. With the MFFE, the receivers were planned to cover $1421 \mathrm{MHz}$ with $\sim 250 \mathrm{MHz}$ tuning bandwidth. This frequency range is well outside the bands exclusively allocated to the radio astronomy service by the International Telecommunication Union (ITU) and in practice is heavily in use by television broadcast transmitters and the then upcoming mobile phone (GSM) networks. Another technical issue was to develop compact feed systems to cover these bands. The size of feed systems more or less scales with wavelength and the available space in the WSRT antennas was rather limited. Astronomers were also unwilling to accept a performance degradation, and, in fact, desired improved performance in all the existing WSRT bands. As frontends around $610 \mathrm{MHz}$ (combined with the $6 \mathrm{~cm}$ receiver) and later also at $327 \mathrm{MHz}$ were added to the receiver suit, this led in the end to the decision to have all these frequencies available in the new MFFE and then eliminate entirely the old receiver sets. Two new receiver bands covering the ranges $250 \mathrm{MHz}$ to $460 \mathrm{MHz}\left(\mathrm{UHF}_{\mathrm{bw}}\right)$ and 700 to $1200 \mathrm{MHz}$ $\left(\mathrm{UHF}_{\text {high }}\right)$ were added to explore the lower frequency universe in the Northern skies as in the south the Indian GMRT came into operation. In response to the VLBI community, the decision was made to add a $13 \mathrm{~cm}$ receiver band to the MFFE to bridge the gap between the existing $18 \mathrm{~cm}$ and $6 \mathrm{~cm}$ bands. This of course added to the complexity of the MFFE design even further!

A good summary of the WSRT upgrade from a science point of view with more details about the background of the MFFE technical requirements is provided Table 1: MFFE perfor- in NFRA note 571 "Thoughts on upgrading the WSRT" by Ger de Bruyn. The mance summary most essential specifications are shown in Table 1.

\begin{tabular}{cccc}
\hline Wavelength & Frequency coverage & System temperature & IF Bandwidth \\
\hline $92 \mathrm{~cm}$ & $310 \mathrm{MHz}-390 \mathrm{MHz}$ & $75 \mathrm{~K}$ & $10 \mathrm{MHz} / 80 \mathrm{MHz}$ \\
$49 \mathrm{~cm}$ & $540 \mathrm{MHz}-610 \mathrm{MHz}$ & $110 \mathrm{~K}$ & $10 \mathrm{MHz} / 80 \mathrm{MHz}$ \\
$21 \mathrm{~cm}$ & $1200 \mathrm{MHz}-1450 \mathrm{MHz}$ & $27 \mathrm{~K}$ & $160 \mathrm{MHz}$ \\
$18 \mathrm{~cm}$ & $1590 \mathrm{MHz}-1750 \mathrm{MHz}$ & $26 \mathrm{~K}$ & $160 \mathrm{MHz}$ \\
$13 \mathrm{~cm}$ & $2215 \mathrm{MHz}-2375 \mathrm{MHz}$ & $58 \mathrm{~K}$ & $160 \mathrm{MHz}$ \\
$6 \mathrm{~cm}$ & $4770 \mathrm{MHz}-5020 \mathrm{MHz}$ & $55 \mathrm{~K}$ & $160 \mathrm{MHz}$ \\
$3.6 \mathrm{~cm}$ & $8150 \mathrm{MHz}-8650 \mathrm{MHz}$ & $90 \mathrm{~K}$ & $160 \mathrm{MHz}$ \\
$\mathrm{UHF}_{\text {low }}$ & $250 \mathrm{MHz}-460 \mathrm{MHz}$ & $175 \mathrm{~K}$ & $10 \mathrm{MHz} / 80 \mathrm{MHz}$ \\
$U_{U_{\text {high }}}$ & $700 \mathrm{MHz}-1200 \mathrm{MHz}$ & $100 \mathrm{~K}$ & $10 \mathrm{MHz} / 80 \mathrm{MHz}^{*}$ \\
\hline
\end{tabular}

* bandwidth remotely selectable

\section{Fitting the operational constraints}

Coming up with a design concept meeting the key specifications listed in Table 1 was only one part of the development challenge. The engineering constraints imposed by the requirement that the MFFE should be compatible with the existing system infrastructure at the WSRT were also a challenge. Arie Hin $\uparrow$ then system engineer at the WSRT and amongst the pioneers of Dutch radio astronomy (see his obituary from the archive of the AJDI200117) considered the design challenges in a very comprehensive overview in his ASTRON Note 516 “Aspecten WSRT 'Multifrequency Frontends'” and in note 539 “'Westerbork'-feasibility aspecten MFFEs", both written in Dutch. Some of the more major technical design constraints are worth noting!

\section{The WSRT Antenna configuration}

Designed for $21 \mathrm{~cm}$ observations, the relatively coarse mesh size of the telescopes in the $3.6 \mathrm{~cm}$ band contributes about $50 \%$ increase of the noise level. Also, the surface roughness at these short wavelengths reduced the aperture efficiency with negative impact on the system sensitivity. Some research had been done in the past (see Insert on Closing the Holes) and a further review led to the final decision that upgrading the reflector surface was not feasible due to mechanical constraints, wind load and weight, and associated costs for improvements.

The short ratio of focal plane length to antenna diameter of $\mathrm{f} / \mathrm{d}=0.35$ required wide feed patterns. With more space available, it is generally easier to design high performance, broadband feeds for a somewhat higher f/d ratio. On the positive side it allowed the re-use some of the existing WSRT feed designs.

\section{Frontend size and mass restrictions}

Mostly for financial reasons, a major modification of the existing primary focus box on the WSRT antennas was not an option. This meant that the new MFFE should fit into the roughly one cubic meter space available. Some ingenious solutions discussed in following sections made this possible.

First of all, the maximum frontend mass needed serious attention. Thus far the heaviest frontends were the cryogenically cooled $21 \mathrm{~cm} / 6 \mathrm{~cm}$ frontends weighting about $275 \mathrm{~kg}$. To accommodate these older frontends the WSRT antenna counter weights already had been strengthened because of the increased stress on the tubing of the support structure. Also, the higher mechanica stress raised some concerns about metal fatigue. It was therefore essential for the MFFE to remain within the $275 \mathrm{~kg}$ maximum weight limit. Combined with the volume restrictions, the weight limit inspired an innovative design for all of the nine receiver bands to fit into each MFFE. 
Westerbork for Cosmology; removing the Bottleneck with the IVC and more

While the work on the wideband digital backend (DZB) and the versatile MFFE receivers was progressing both of equal frequency bandwidth, the connection between the two turned out to be an unplanned bottleneck Cer de Bruynt wrote a proposal to NWO on "Cosmology with the WSRT" making the case for using the full bandwidth capabilities of the new MFFE and digital backend. Now funding was available to remove the bottleneck!

The IF-to-Video Converter (IVC) would be the new link between the MFFE and the DZB. A main feature of the IVC was its capability to process 32 channels with $20 \mathrm{MHz}$ bandwidth of the $160 \mathrm{MHz}$ IF bandwidth from the 14 telescopes plus two spare inputs. This led to a system with 256 converter units with associated modules like the Local Oscillator (LO) and controls. All the IVC hardware was fit into 5 large 9-inch system racks.

First custom analog IC in R.A.?

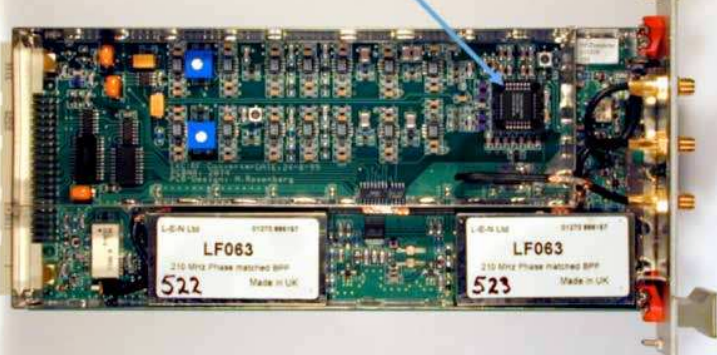

The frequency conversion board including the first ana log custom Integrated Circuit in BiCMOS technology by Philips in 1997.
Bert Woestenburg was given the challenge to lead the IVC projec which began in 1995. This would be a "high volume" production project and for the very first time, ASTRON decided to outsource design and production to industria companies beginning a new era of industrial partnerships and radio astronomy development. Catena Microelectronics carried out a feasibility study to integrate parts of the IVC functionality in a single chip including digital filtering techniques. However, digital filtering still had some years to go before becoming a competitive alternative to the analog solution.

As always, cost reduction would lead to a performance reduction. Ger de Bruyn then suggested to limit the number of filter channels to 4 without sacrificing too much on the science capability. Tough requirements in a harsh environment of radio interference was not the only challenge. Amplitude and phase accuracy and stability were equally important.

The feasibility study in 1997 recommended the development of a custom image rejection mixer integrated circuit. This would become the first custom analog integrated circuit used in radio astronomy! The design included a double conversion concept to avoid unwanted LO signals in the pass-band and ASTRON made an order to Caten for the development and proden of the "IVC-mixer" chip.

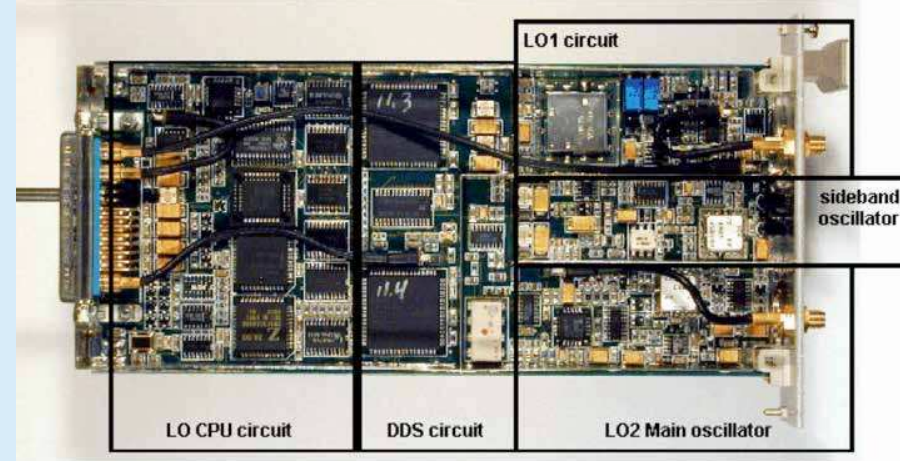

One of the 256 Local Oscillator boards with two conversion stages including $\mu \mathrm{Hz}$ fringe rotation.

Unfortunately, the original idea of a fully integrated on-chip design did not achieve the expected cost reduction, and a plan to outsource the development and production of the various subsystem modules was the next best option. A French company, Tekelec-temex, with experience in custom electronic design was approached to further develop the IVC modules, except for the converter module which was tasked to Lambert Nieuwenhuist' and the converter control and filter module which were handed over to Hans Rosenberg. Teke Tekelec-temex designed and produced excellent cable equalizer units necessary to compensate for the $3 \mathrm{~km}$ cable loss to the telescopes. A narrow band $(200-220 \mathrm{MHz})$ and very steep filter with ultra linear and equal phase relation was another custom designed component. Over 500 filters, each manually fabricated and tuned to the correct high filter requirements, were ordered from the UK company L.E.N.The local oscillator system remained an unsolved problem by Tekelec-temex, and so the IVC LO-system came back to the ASTRON design table. The major issue in the design was achieving the phase accuracy, stability, and noise within a compact module, and gen- erating two frequencies for the converter unit. Fringe correction was one of the LO-module features and required a 14 micro Hertz step of the master oscillator. Phase stability over temperature was another difficult requirement, and it had to be addressed in all aspects of the IVC project, including development, production, installation, and ultimately, system maintenance. Nico Ebbendorf and Arduin Eybergen developed the LO concept while Arie Doorduin

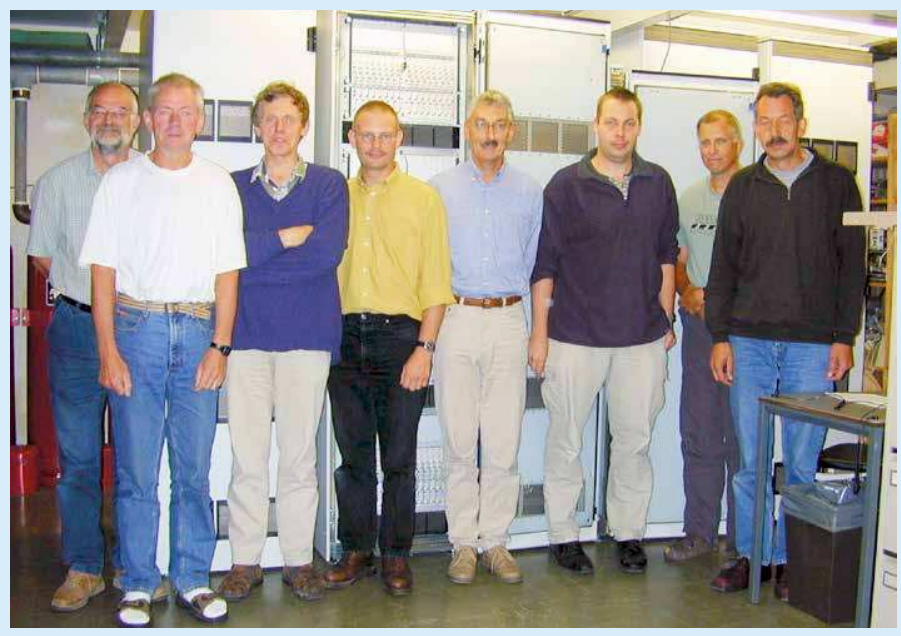

The proud team formally handing over the "cosmology" system to the Observatory. It allowed to use the full capabilities of the new Multi Frequency Front Ends as well as the new DZB backend correlator system. From left to nigh. Hans Weggem ans, Lambert Nieuwenhuisy, Albert Bos, Nico Ebbendorf, Bert Woestenburg, Mark Bentum, Rob Millenaar and Kees Brouwer 
Signal distribution between MFFE and central building

Two types of critical signal transport between the antenna mounted frontends and the central system are:

- Distribution of a common reference frequency from a central master clock to each of the frontends, and

- Transport of the received astronomical signals to the common correlator.

At the time of development of the MFFE, optical fibre transmission systems were not mature enough to fit the stringent phase/delay stability requirements for the WSRT. Therefore, it was decided to use the existing, proven, coaxial cable network. These $3 \mathrm{~km}$ long coaxial cables, buried under ground and constantly flushed with dry nitrogen gas, had a proven excellent stability. The only remaining challenge was the rather high, frequency dependent loss over the larger bandwidths to transport the astronomical signal from the frontends to the central correlator. This compensation was done in the equalizers of another system (see Insert on Westerbork for Cosmology).

\section{Streamlining WSRT operation}

From an operational point of view, the capability of rapidly changing to another frequency band in the new frontend was the major design driver. As the previous frontends mostly covered only one wavelength band, it was necessary to change the frontend on all telescopes to switch to another wavelength. The burden of this process could take as long as two weeks depending on the type of frontend involved. Apart from the huge operational load, the advent of the Very Long Baseline Interferometry (VLBI) to the WSRT turned this into a serious problem. VLBI observations are normally organized as campaigns that last one or two weeks several times a year. During such a campaign, a number of different wavelengths were required and in practice this would limit the use of the WSRT. The new MFFE design had to overcome this problem which was reducing WSRT participation in VLBI.

Radio astronomy was facing an increasing level of interference, for which the MFFE frequency agility proved extremely beneficial. It allowed rapid changing from one frequency to another which was free of interference.

The increasing (level of) interference had two major causes. First, the nineties showed a dramatic increase of spectrum usage, especially in the range up to $2 \mathrm{GHz}$ which was important for astronomy. This development directly affected radio observations, even in the frequency bands protected by international treaties under ITU supervision. For example, there is interference from GLONASS, the Russian counterpart of the Global Positioning System, and later, the global communication system Iridium. Titus Spoelstra $†$ (see also the contribution in the Chapter covering Ionosphere) who then operated as frequency manager for radio astronomy, provided an overview of the spectrum allocations versus the MFFE receiver bands in NFRA note 606 "Multi-frequency frontends: frequency coverage and frequency allocations"

There was a growing demand to observe outside the protected radio astronom ical frequency bands. The observation of neutral hydrogen at large red shift in the range from approximately $250 \mathrm{MHz}$ to $1400 \mathrm{MHz}$ is just one important example. Extensive spectrum monitoring at the WSRT site showed that some parts of this range are sometimes usable for radio astronomy when interfering signals are below a certain level. This depends not only on the actual occupation of it by the legitimate user but also on propagation conditions, making the availability of this spectrum very dynamic

Finally, the simplified operation of a single MFFE for all telescopes instead of five different receivers had other advantages. The number of spare parts in stock was reduced, and there was a reduction of labour involved in service and maintenance. It also overcame the cumbersome situation of replacing a cooled frontend operating at $21 \mathrm{~cm}, 18 \mathrm{~cm}$ or $6 \mathrm{~cm}$ for an uncooled, less sensitive frontend, in one telescope in case of failure. This event happened regularly and would seriously affect the quality of observations.

\section{MFFE Design Concept}

Taking the requirements and constraints as described previously the MFF project team started to investigate the various design concepts. This activity led to a design concept for the new frontend which was described in NFRA Note 555 "Report Feasibility Study Multi Frequency Frontend" by Gie Han.

The picture in Figure 1, shows an extremely early MFFE version brought from Spain and happily unpacked by Jaap Bregman and Bert Woestenburg on the traditional Dutch Sinterklaas day around 1990 in the cantine of the old building. While Gie Han lead the overall project, Jaap was responsible for the thermal design and Bert Woestenburg for the team leading the development of the Low Noise Amplifiers.

Published in March 1990, Note 555 was thoroughly reviewed by an astronomy expert panel and external receiver engineers. Around that time it was also decided that Richard Strom as staff astronomer would be the Project Scientist on behalf of the user community and

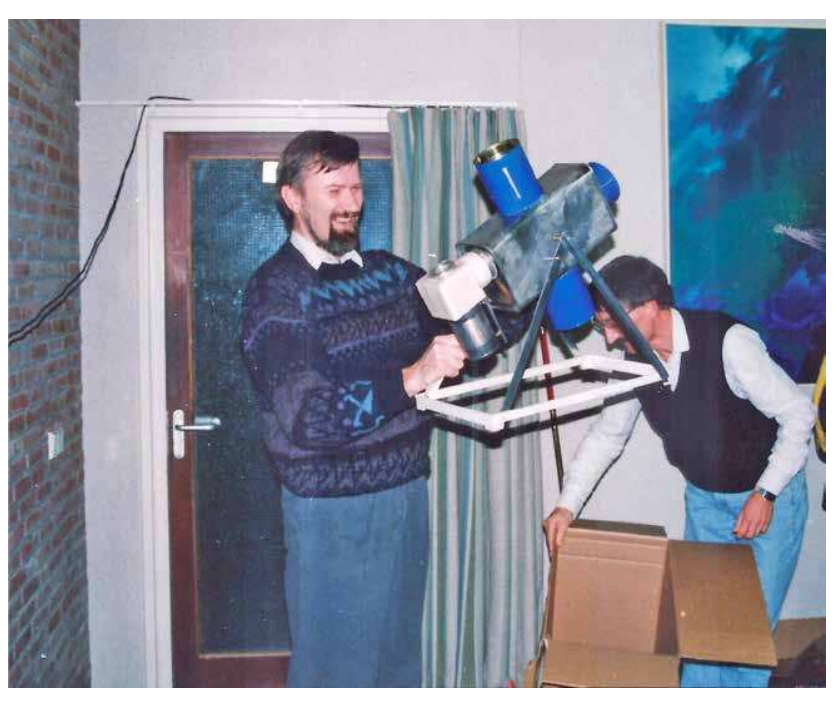




\section{Mechanically Rejuvenating the WSRT}

Repainting

By 1992, the paint of all steelwork for the 14 WSRT telescopes was in poor condition. A detailed report listed all the maintenance necessary, and it would cost over 3 million Dutch guilders (nearly 1.5 million Euro). Only half of these costs could be funded so WSRT director Hans Kahlmann made the decision to do half the job with premium quality rather than doing the whole job cheaply. We would start with the most critical telescop elements.

The primary reflectors were the priority. While in the 1975-1976 period the originally glued stainless steel mesh to the 98 panels of each reflector was fixed with self-drilling screws and now seemed fine, the new problem was with the corrosion of the frames which have a wall thickness of only $2 \mathrm{~mm}$. On top of that, it also appeared that the mesh was attached to the frame on the rim but nowhere else, and permitted the centre area to from the parabolic shape. This was confirmed by holography measurements by Hans van Someren Greve. The interferometric surface area measurements accurately identify locations that deviate from the ideal parabolic shape.

In a most daring enterprise starting in 1994, the first reflector (RadioTelescopes or RT5) was lifted from the supporting structure and transported to the old assembly hall. To preserve the correct reflector shape, only the even numbered panels were removed for sandblasting, painting and refitting. Blind rivets were dipped in paint and inserted in the holes, replacing the old self-drilling screws. Next the second, odd numbered half of the panels were taken out for refitting. New holography measurements confirmed that the reflector area performed equally well to the original parabolic shape whose accuracy was about $1.4 \mathrm{~mm}$ over the $25 \mathrm{~m}$ diameter when measured in 1977 by Arnold van Ardenne.

For the retrofitting of the other reflectors, a plan was made for a smaller operating array of 13 telescopes for the next 3 years. The scopes for the next 3 years. The 1996 for revision, and RTo would remain without reflector for a 3 year period. Every 12 weeks a refitted reflector was transported to the next
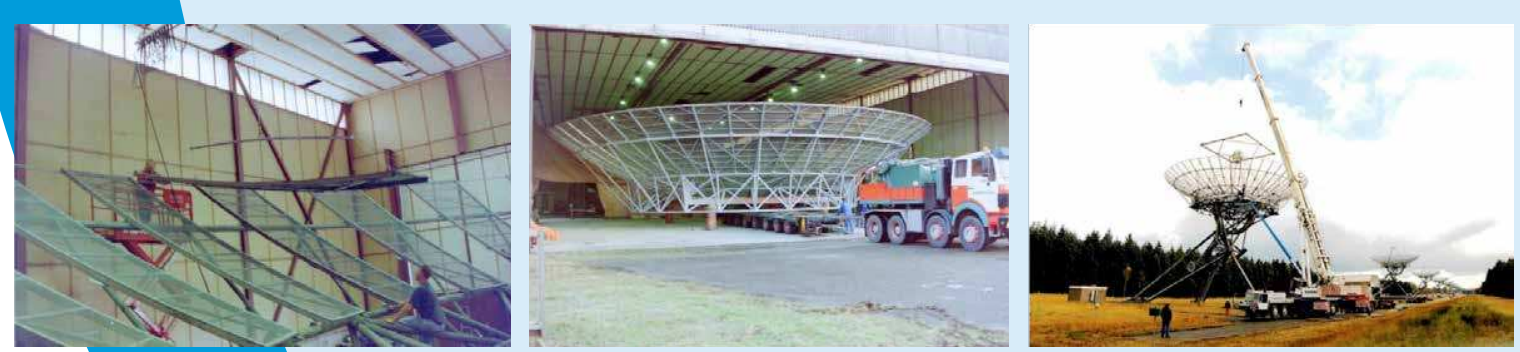

Left to right: 1994 removal of outer-panel of RT5. 1994 reflector ready for transport to RT5. 1998 November, lifting of last revised reflector on RTo

telescope and interchanged within 3 weeks. The whole operation ended on December 1998 when the last reflector was lifted onto telescope 0 . Reinforcing the Counter Weights A new observing mode called "mosaicking" required rapid alternating on-off source measurements over a prolonged period. This put a lot of mechanical stress on the telescopes during the continuous 8-12 hour observations and the situation became even more serious after 2000 when the new Multi Frequency Front Ends started their observing life on the telescopes. They each weigh $250 \mathrm{~kg}$ !

The electrical drive can accelerate from $1.4 \mathrm{~Hz}$ to $100 \mathrm{~Hz}$ in 3 seconds but the 30 tons of counter weight. dangling a good $7 \mathrm{~m}$ from the polar

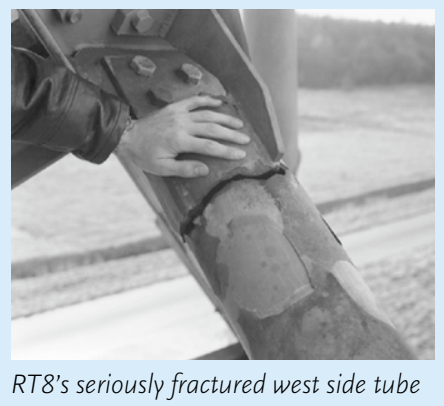

Upon further inspection all the ther telescopes showed cracks on his same location, sometimes as ong as $8 \mathrm{~cm}$. Additional steel reinforcement was subsequently welded on all tubes of the counterweight construction, while a hydraulic jack under the counter weight relaxed the steel members during the reinforcement.

The WSRT telescopes operated without further problems for almo ten years, including 4 years with

of the hour-angle counterweight in 1995 the heavy MFFE's, but then a loud

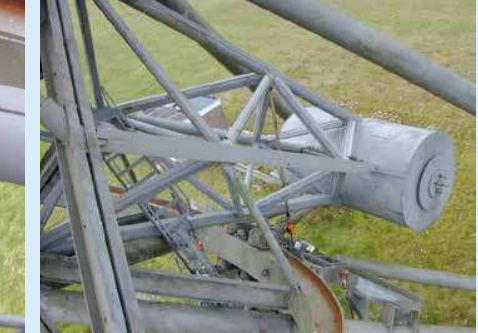

strengthening member added. Right: four angaired tube with a construction, with all members doubled in steel on all

Thesacking obsening mode caused metal fatigue and finally factured side tube on RT

noise was heard in 2004! It turned out that the transition from circulair tube to the flat connector plate needed repair (see picture). We were able to add another reinforcement plate but the mosaic measuring mode continued to attack the stiffened construction, and in mid 2005 we found cracks in the steel at the end of our reinforcements that had been made in 1995. Additional steel was welded on this weak spot (see picture on the right) found on 12 telescopes, and so far no more cracks have been noticed. For the new Apertif era, the time to reach full speed has been increased to 5 seconds which will reduce the acceleration.
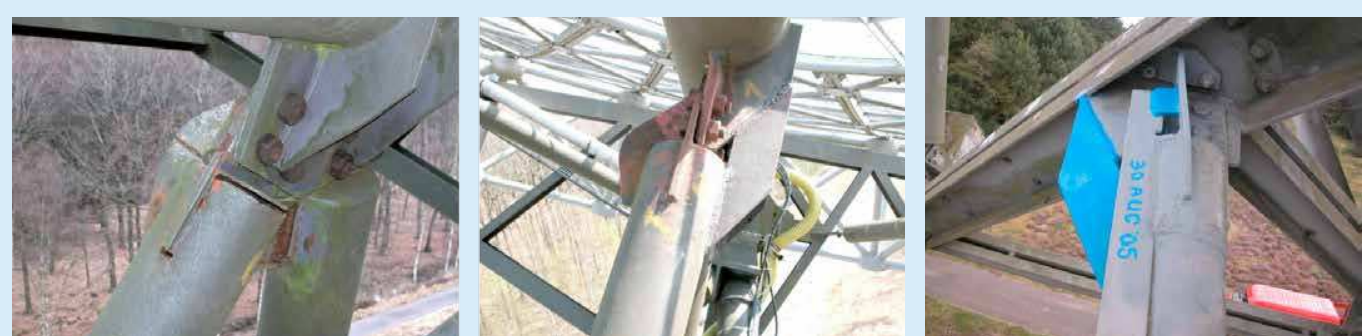

Left: RTC in 2004 connection to declination axle is completely broken. Middle: Connection to the declination axle, re-welded and reinforced on all 14 telescopes. Right: additional strengthening of the tube counterweight construction now on 12 telescopes 
the WSRT. The MFFE design concept was positively reviewed by the panel and supported the decision to proceed to the detailed design and construction phases.

\section{Developing for the MFFE}

Starting the developed phase formally then allowed a larger team to work on the MFFE, and in the electronic labs, the emphasis was on MFFE up to 1997. The following is a condensed description of the MFFE design.

Receiver electronic description

All MFFE receivers are electronically based on the double super heterodyne principle. For a single polarization channel at each observing band, a brief description is as follows

The incoming astronomical signal focused by the parabolic reflector enters the feed and is split into its two orthogonal components by an Ortho-Mode Transducer (OMT). Between feed and OMT a noise signal can be injected through a directional coupler for level and polarization calibration.

Most of the OMT's, transfer relays, and LNA's that operate above 1.2 GHz are in the cryostat of which the inner part is cooled down to a physical temperature of approximately $20 \mathrm{~K}$ (equivalent to $\sim 253^{\circ} \mathrm{C}$ ) to reduce their noise contribution.

After the LNA follows a RF band-pass filter. This filter suppresses the unwanted image frequency and multiple responses from the first mixer. Before the first frequency-down conversion the signal passes a $180^{\circ}$ phase modulator. Together with a demodulator in the backend, this enables a further suppression for any possible interference.

A tunable, synthesized oscillator provides a Local Oscillator (LO) signal for the first mixer. The frequency synthesizer is tunable in $1 \mathrm{MHz}$ steps.

The first Intermediate Frequency (IF) is centred on $1 \mathrm{GHz}$ and the IF band-pass filters have a bandwidth of either $160 \mathrm{MHz}, 80 \mathrm{MHz}$ or $10 \mathrm{MHz}$. These bandpass filters provide the main selectivity of the receivers and set the maximum instantaneous bandwidth for further processing.

A fixed, $900 \mathrm{MHz}$, phase locked synthesizer provides the second local oscillator signal. It is used to convert the signal to the second IF frequency around $100 \mathrm{MHz}$ using only low-pass filters which are adequate to suppress the unwanted sideband. After further amplification, the received signal is sent to the central back end by coaxial cable for signal processing.
By injecting a known power level between feed and LNA the absolute powe level calibration of a receiver channel is measured. This power is generated by a broadband noise source with known level and can be switched on and off to make a comparison measurement. Consequently, the power level of the incoming astronomical signal is measured.

Polarization calibration for precise Stokes parameter measurements requires the comparison of orthogonal polarization channels in a receiver with each other to resolve the differential offsets in gain and phase. In the MFFE, two methods are used to determine possible offsets.

In the first method, the same signal is injected into each orthogonal polarization channel. Measured output differences characterize the offsets. In this method, the signal from the noise source for the power level calibration, is used. Depending on the selected receiver, the two identical signals to be injected in each of the two orthogonal polarization channels are obtained through a power splitter. For the waveguide systems above $1.2 \mathrm{GHz}$, the noise is injected into a circular waveguide at an angle of 45 degrees relative to the orthogonal, propagating the $\mathrm{TE}_{11}$ waveguide mode.

The second method of polarization calibration uses the transfer relay between the outputs of the OMT and the LNA of the orthogonal polarization channels By making through and cross measurements and comparing the two results, the offsets in gain and phase can be determined. As the signal paths before the noise injection point and the transfer relay cannot be calibrated, the receiver design should take care such that these are identical for both orthogonal polarizations.

Signal levels and gain distribution in the receivers are chosen in such a way that there is minimal internal intermodulation.

All $\mathrm{LO}_{1}$ and $\mathrm{LO}_{2}$ synthesizers are locked to the two reference frequencies, $1 \mathrm{MHz}$ and $180 \mathrm{MHz}$, fed into each receiver by a single coaxial cable, and derived from the central frequency standard in the main building. A diplexer inside the MFFE splits the two reference frequencies and distributes them among the synthesizers.

This first local oscillator is an indirect phase locked loop synthesizer. A custom made YIG tuned oscillator is used to achieve a wide tuning range combined with high spectral purity. A sample of the output signal is down-converted by a sampling mixer, sent to a programmable digital divider and compared with the $1 \mathrm{MHz}$ reference frequency. The sampling mixer is driven by a second synthesizer operating in the frequency range $180 \mathrm{MHz}-183 \mathrm{MHz}$ and phase-locked to the $1 \mathrm{MHz}$ and $180 \mathrm{MHz}$ references. The design is such that phase coherency is ensured between all MFFE. 


\section{Merely a Filter?}

"It doesn't work!", the WSRT telescope engineer Harm-Jan Stiepel said, when dropping a set of neat blinky filters on my workbench. This is not a comment you want to hear from a colleague, especially if you have just started your career as an $\mathrm{RF}$ engineer

But let's start at the beginning of this story (and to give this away for the impatient reader: They actually worked!) In 1998 we rolled out 16 of the wonderful new Multi Frequen Front Ends (MFFE) at Westerbork. During the qualification tests some strong unexpected signals appeared in the $92 \mathrm{~cm}, 49 \mathrm{~cm}$ and UHF-High bands. It appeared that in August 1997 the regional television service, RTV-Drenthe, started analog broadcasts from the Smilde tower which is located only 13 kilometres away from the WSRT. The carrier frequency was $503.25 \mathrm{MHz}$. Immediately, Mark Bentum - the

telescope scientist at the WSRT found that the $503.25 \mathrm{MHz}$ mixes with the $182.25 \mathrm{MHz}$ carrier of the Nederland I service and provokes an intermodulation product at $321 \mathrm{MHz}$ which exactly falls in the MFEE $92 \mathrm{~cm}$ receiver band and was the probable cause of the strong unexpected signals.

The above mentioned new engineer was charged to build a set of filters that suppressed the interference and had to be placed in front of the balanced low noise amplifier that was generating the intermodulation product at $321 \mathrm{MHz}$ due to its nonlinearity. Leo van de Ree and Lambert Nieuwenhuis were in charge of the $49 \mathrm{~cm}$ and UHF-High solution respectively.

I digged into filter synthesis, simulated, designed, prototyped, tuned optimized, re-designed and reprototyped a bandpass filter. At the meof the low loss filter. The red line shows the the suppression of the unwanted 503.25 MHz and $182.25 \mathrm{MHz}$ sigand $182.25 \mathrm{MHz}$ signals (Marker 1 and while the loss inside band is only a tenth band is only a tenth of a Decibel (2\%).
Jürgen Morawietz

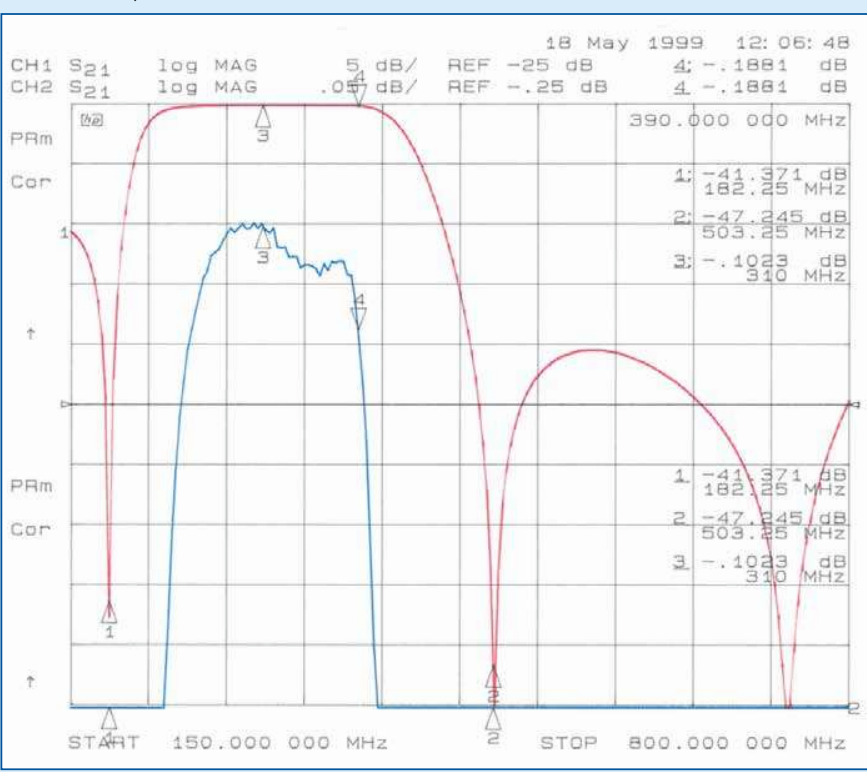

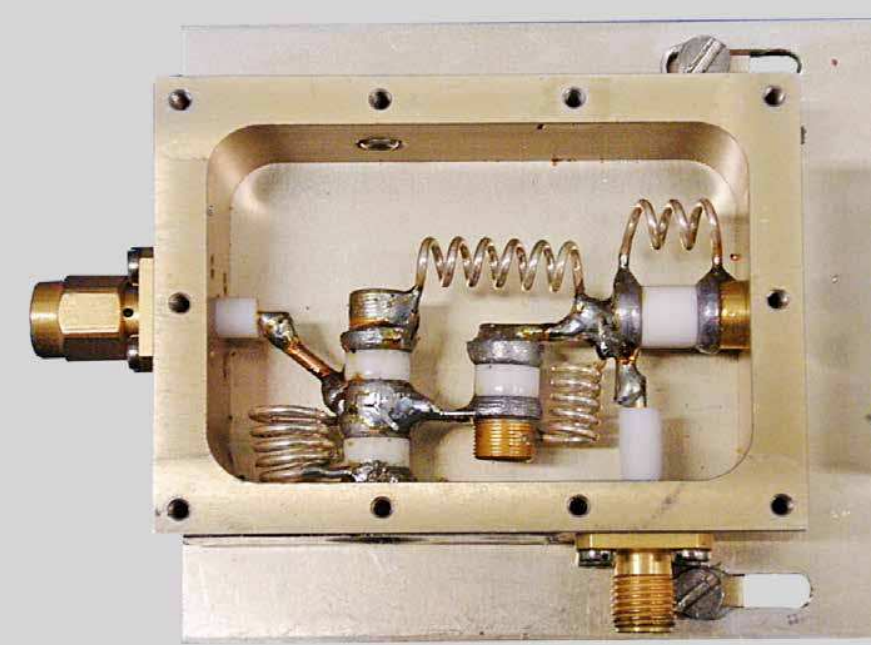

Figure 2: The filter for the Multi Frequency Front Ends. ã Jürgen Morawietz

neat aluminum enclosure and I had my filters ready for testing. I would impress my boss and project leader Bert Woestenburg!

The implementation and tests of this prototype in the MFFE ended up with the disastrous message that began this story, but SParameter measurements on the filters showed that they had the specifice istics and should have worked!

Together with Harm-Jan and Mark we started an extensive investigation the intermodulation products in the MFFE. Even nonlinearities on passive components due to transition between different metals or bad connections - which is known to appear in high power RF systems - were considered. Nothing was found.

The conclusion was that we have a perfectly working MFFE (including filters) and the only solution was that the intermodulation was not very friendly and interested techni- that a large part of northern Netherlands couldn't watch television for some hours during the installation of these filters.

New qualification tests were carried out with an MFFE in the Westerbork telescopes. It performed well and the interference disappeared. Problem solved! So finally, series production of 64 filters could start, all crafted and handtuned by Anne Koster. The rookie engineer vindicated himself and 20 years later he's still a proud - and now a bit greyed - member of the ASTRON staff. He's designed many filters - some of them even located behind the moon But that's another story.

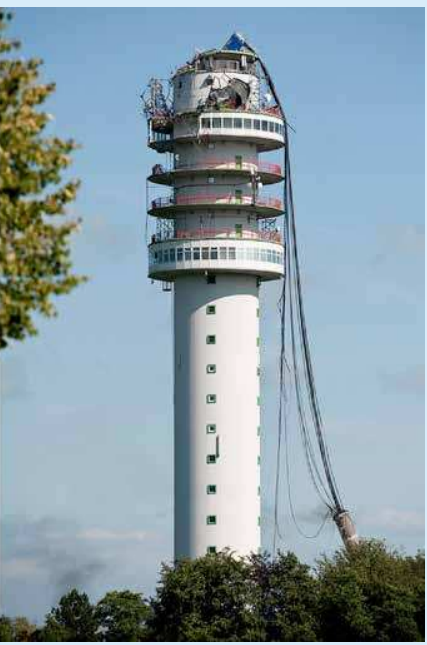

Figure 3: The unfortunate and collapsed transmission tower in Hoogersmilde on the afternoon of July, 15th 2011. Obviously, no radio interference was noticeable afterwards! (c) Jürgen Morawietz cians to do some measurements on the high power UHF transmitters.

We confirmed that the intermodulation was generated in the transmitter. We were even more surprised when they offered to install a filter at their expense! We hereby apologize
Actually there was one other option that was demonstrated some years later when the Smilde tower caugh fire and collapsed. I swear we have nothing to do with this incident 
A single embedded microcomputer in the MFFE control system controls and monitors all functions in the sub-systems. The control system also handles the interface between MFFE and the central WSRT computer. However, the real time functions like $180^{\circ}$ phase switching and switching the noise sources are directly controlled through separate hardware lines from the central control logic

Finally, auxiliary systems include the temperature control circuits of the MFFE and the feed rotator drive system.

Using the MFFE system allows for simultaneous observations at the following wavelength combinations:
1. $92 \mathrm{~cm} / 3.6 \mathrm{~cm}$
5. $\mathrm{UHF}_{\text {low }} / 6 \mathrm{~cm}$
2. $13 \mathrm{~cm} / 3.6 \mathrm{~cm}$
6. $\mathrm{UHF}_{\mathrm{low}} / 18 \mathrm{~cm}$
3. $49 \mathrm{~cm} / 6 \mathrm{~cm}$
4. $\mathrm{UHF}_{\text {low }} / 3.6 \mathrm{~cm}$
7. $\mathrm{UHF}_{\text {low }} / 21 \mathrm{~cm}$

The possibility of dual band observations became fully operational with the advent of the DZB back end. However due to limitations in the coaxial transmission system between frontends and back end, dual band observations allows for only one polarization channel per band.

\section{Mechanical design}

As for the previous WSRT frontends, a support frame was designed and constructed of hollow metal tubes with square cross section. Figure 2 shows a 3-dimensional drawing of this frame.

The front of the frame (left hand side in the figure), is closest to the phase center of the feed. It is mounted on a support structure in the frontend box of the telescope with precision pins. In this way, the mounting results in the position

Figure 2: A 3-di-

mensional drawing

(see text).

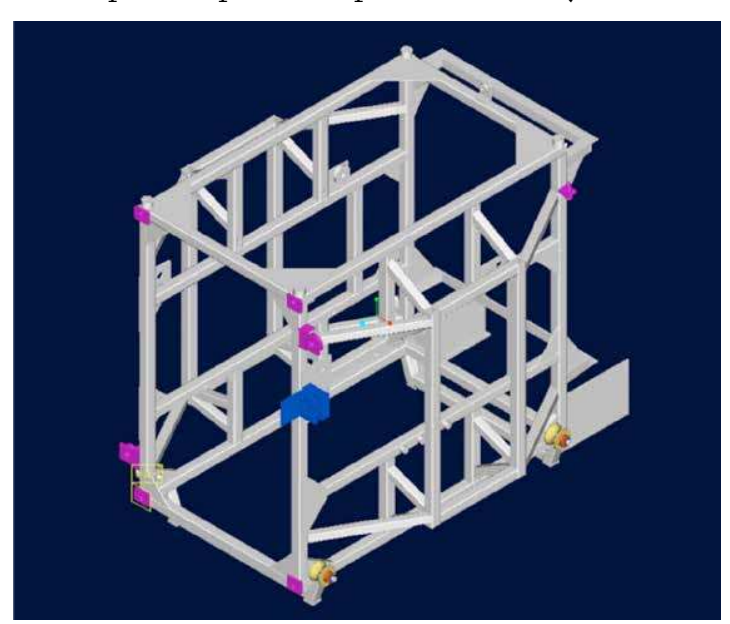

of feed phase centre located precisely with the focal point of the parabolic reflector.

ther main requirements of the mechanical construction were (i) to accommodate all necessary sub-systems in the frame. This was in particular challenging for a proper functional placement of the feed systems, (ii) Overall weight lower than 275 kilograms, (iii) Maintain a maximum deviation of approximately $1 \mathrm{~mm}$ in both lateral and axial directions between the feed phase center and the focal point of the parabolic reflector for any telescope position. This is to avoid too much antenna gain reduction due to phase errors for an axial displacement and excessive beam squint for a lateral displacement. To fulfil the first requirement a construction was chosen where a rectangular box was used for the cryostat. Figure 3 shows a view of the single and completely mounted cryostat for all cooled observing bands in an MFFE including the cooled LNA for each band in two polarizations. The many windows to the cryostat required a thorough thermal design analysis which was done by Jaap Bregman.
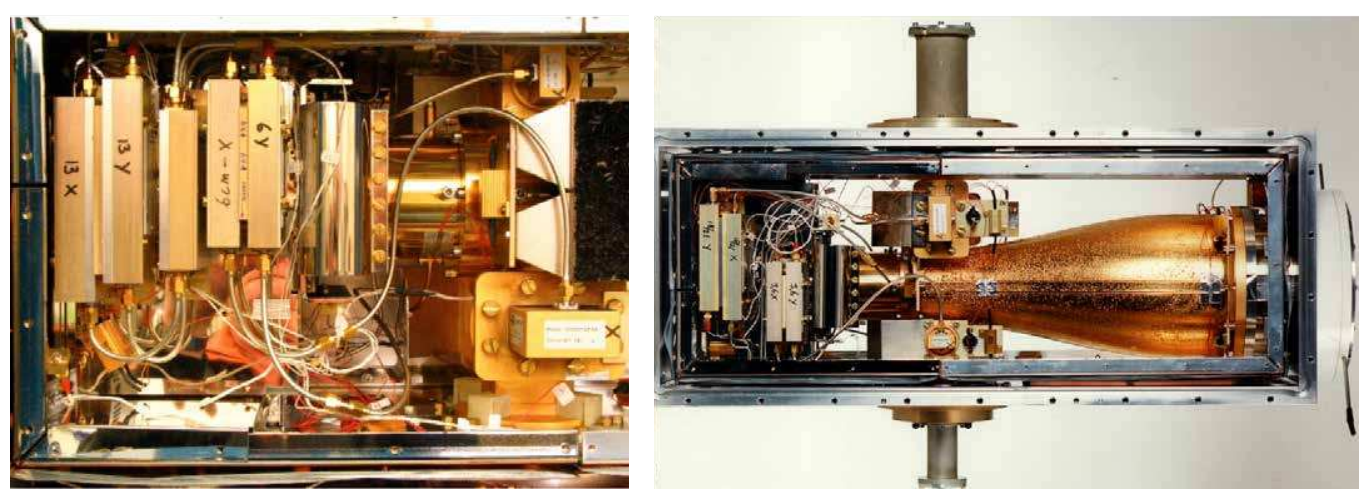

By rotating the whole structure inside the frontend frame, each feed system can be placed in the feed focus. The picture in figure $4 \mathrm{a} / \mathrm{b}$ gives an impression of this construction, which as a whole fits inside the focus box.
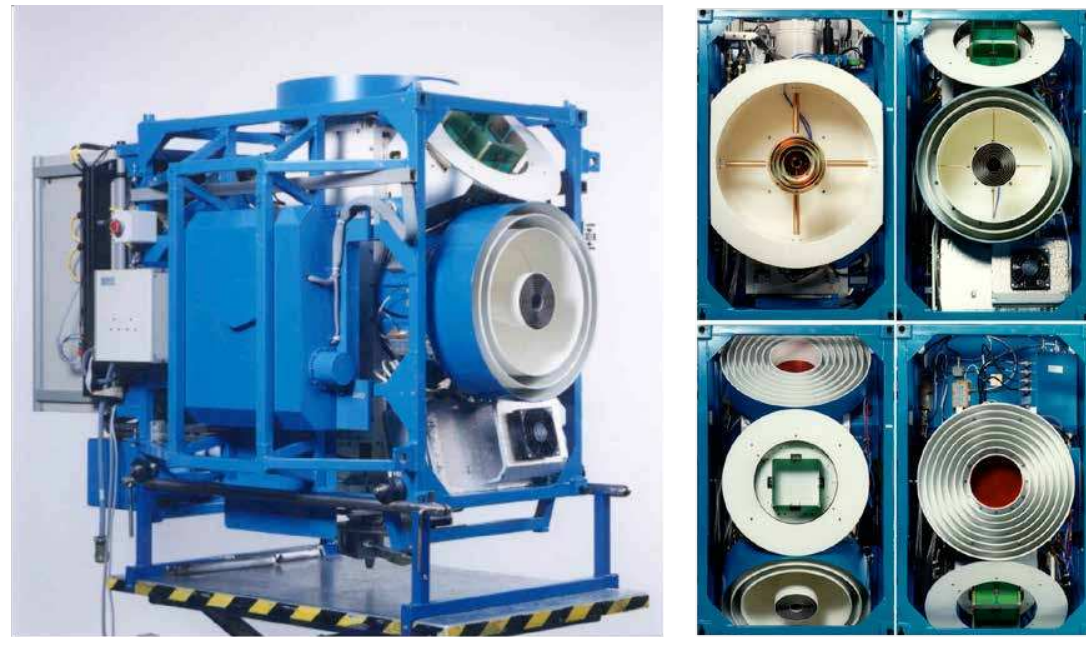
through a completecryostat of the MFFE cooled section. All four cooled receivers are mounted in this cryostat for two polarizations. The $18 / 21 \mathrm{~cm}$ signals enters from the right, the $6 \mathrm{~cm}$ lof the $6 / 50 \mathrm{~cm}$ combined feed) from below and the $13 / 3.6$ $\mathrm{cm}$ (of the $92 / 13 / 3.6$ cm triple feed) from the top. On the left the cooled amplifier arrangement shown in more detail

Figure 4: Left: Overall MFFE construction with rotatable feedsystems around a single cryostat. Right: Looking into the feed systems rotated for each position; top-left $92 / 13 / 3.56 \mathrm{~cm}$, top-right Right: Looking into the feed systems rotated for each position; top-left $92 / 13 / 3.56 \mathrm{~cm}$, top-right $50 / 6 \mathrm{~cm}$, bottom-left UHF high, bottom-right $21 / 18 \mathrm{~cm}$. Both UHF high and
folded dipoles of which the UHF low is fitted on the telescope frontend box. 
The requirements concerning frontend weight and stiffness were extremely hard to combine. An extensive use of aluminum instead of steel as was used in older frontends, resulted in a major weight reduction. This was made possible by the first time use of advanced mechanical 3D CAD tools in combination with finite element programs to simulate the static behavior of mechanical structures. It made it possible to optimize the geometry and minimize the material weight for given mechanical specifications. This resulted in the rigid frame construction shown in Figure 4. A special high strength aluminum alloy, $\mathrm{Al} 7075 \mathrm{~T} 6$, is used for the square tubes to guarantee the set requirements.

Using these exotic materials without deterioration of their properties needs special skills and knowledge. While the prototype was built by the NFRA mechanical workshop by Jan Idserda, the series production was contracted out. A small Dutch company who was specialized in building aluminum chassis for racing cars built all the production frames for the MFFE. Their expertise in this area resulted in familiarity in working according to standards of high accuracy and a capability in special aluminum welding techniques and this contributed to the making of the MFFE frames. Other companies were involved in adapting the antenna boxes for the MFFE on which the air-pressured antenna removal system for $\mathrm{UHF}_{\text {low }}$ was mounted.

A further major weight reduction was obtained due to the use of advanced honeycomb material for some of the cryostat walls instead of solid aluminum ones. Instead of a weight of $13 \mathrm{~kg}$ for one single cryostat lid consisting of solid aluminum, a honeycomb lid weighs merely $2 \mathrm{~kg}$.

\section{Lightning and electromagnetic compatibility}

During the nineties, the WSRT upgrade included increasing the astronomical and reducing the receiver noise by developing cryogenic receivers: The Multi Frequency Front Ends (MFFE). This basically meant replacing all receivers. Since support equipment and systems were nearing end of life, they had to be replaced or refurbished as well.

With all these upcoming changes it was clear that the grounding

lightning protection, and in genera the electromagnetic compatibility situation (EMC) had to be thoroughly reconsidered, and that the do's and don'ts were taken into account in the new designs. It was not only for safety and lightning susceptibility considerations. EMC is also important to ensure that the very weak astronomical signals are not distorted by externally generated not distored by exteraly generated and by selfgenerated interfere, and also to ensure that the time and frequency reference systems can

Figure 1: Floor plan of the WSRT control room, showing cabling ducts of the electron. squares): the advice was not to use a star-grounding pattern, but to create a dense tem, but to create a dense mesh pattern in order to to interference

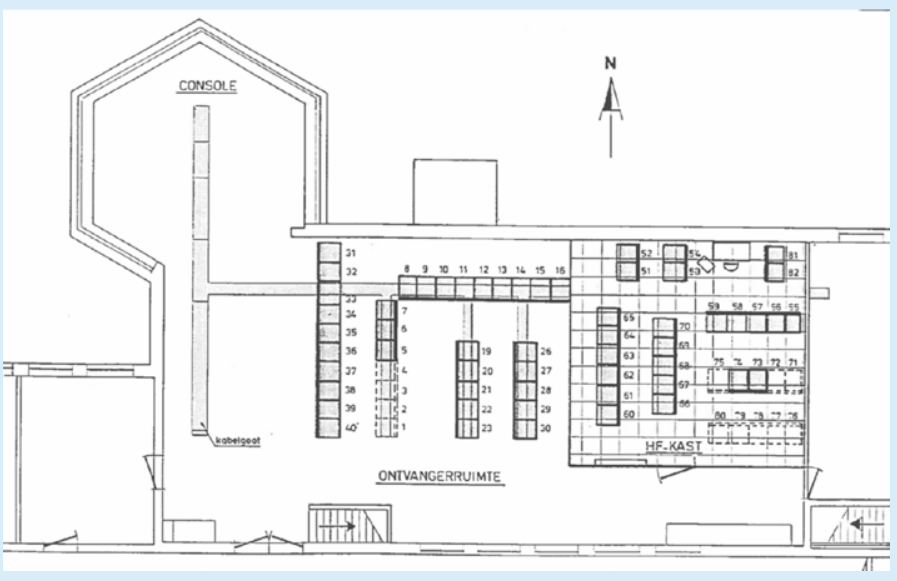

provide stable signals, and meet the geodetic and pulsar timing stability requirement of 0.3 ps per day and $0.3 \mathrm{~ns}$ over years.

In 1992 at the R\&D laboratories, Gie Han Tan started an analysis concerning the lightning protection of the MFFE, and concluded that the best way to protect the receivers was to place them into a fully closed Faraday cage, integrated within the telescope focus box, except obviously for the antenna part. In the design, the control and power lines were to be protected by special filters mounted to the cage wall. Indeed, in the past two decades the currents induced by lightn ing had never damaged the fourteen receivers (frontends), and the new designs had to be at least as robust as the old ones.

In 1996, the systems group at the WSRT looked into the EMC situation of the WSRT as a whole, including the telescope structures, power grid, the control building grounding, and cabling connections to the elescopes. Looking at the relevant EMC norms it all seemed compliant. With the upcoming major changes, and also after a warning shot by a recent lightning strike in a tree near telescope number destroying optical coupling diodes of the telescope control systems, we decided to ask an external expert, DARE Electronics in Woerden, to

screen the WSRT and provide EMC advice

The EMC site survey concluded that the control building lightning protection was ok, that the grounding of the individual telescopes could be improved by adding grounding strips at a few places in order to avoid large lightning-induced currents running over the cabling shields. It was also noted that external cabling entered the control building from different directions. From an EMC point of view it would be best to re-route them to enter at one spot, but as that was ent practical expert advised to connect the cabling shielding to one common grounding strip. All this and some other related advice was relatively easy to implement, so there was ample time for Pieter Donker, Hans Weggemans, HarmJan Stiepel and the rest of the crew to do this, well before the new systems were expected to arrive from the Dwingeloo laboratories.

The company also advised to transconsole roomi of the control building, as these sys. tems were generating radio 'noise' that leaked into the two telescopes closest to the control building. This we already knew, and was part of our planning in extending the Faraday cage for the new equipment.

The modifications turned out to be a great success, allowing a seamless integration of the up coming upgrade equipment. In addition, this EMC work also helped Prof. Howard Reader and Gideon Wiid to prepare recommendations for making the South African

MeerKAT radio telescope lightning proof and RFI robust. 
Thanks to its advanced design, for which a Reliability Availability, Maintainability (RAM) analysis was done, it could now safely be said that the knowledge of building receivers at ASTRON culminated over tens of years in the extremely reliable and agile MFFE which required very low maintenance and had a correspondingly observing efficiency.

\section{Two Phases}

Around 1997 a little ceremony was held to celebrate the first phase delivery of the MFFE (UHFlow / UHFhigh) system. All present remember the act performed by our Board member Renzo Sancisi in ceremonially placing a frontend into the telescope frontend box using the cherrypicker hoisting platform. Renzo's apparent reluctance to join Gie Han Tan and Harm-Jan Stiepel on this high act was however solved. An apparent problem prevented the whole heavy load to bridge the $12 \mathrm{~m}$ up and a load reduction was required. As it happened, Renzo was so kind as to give up his place and to step off the platform to watch the scene from solid ground.

After that, the UHF commissioning phase was successfully done by Richard Strom and reported in Note 654 in 1997

Work continued in the second phase of finishing the whole set of receivers. The picture (Figure 5) at the NAC (Dutch Astronomy Conference) in 1997 shows Lodie Voute, Gie Han Tan and Arnold van Ardenne discussing the pro-

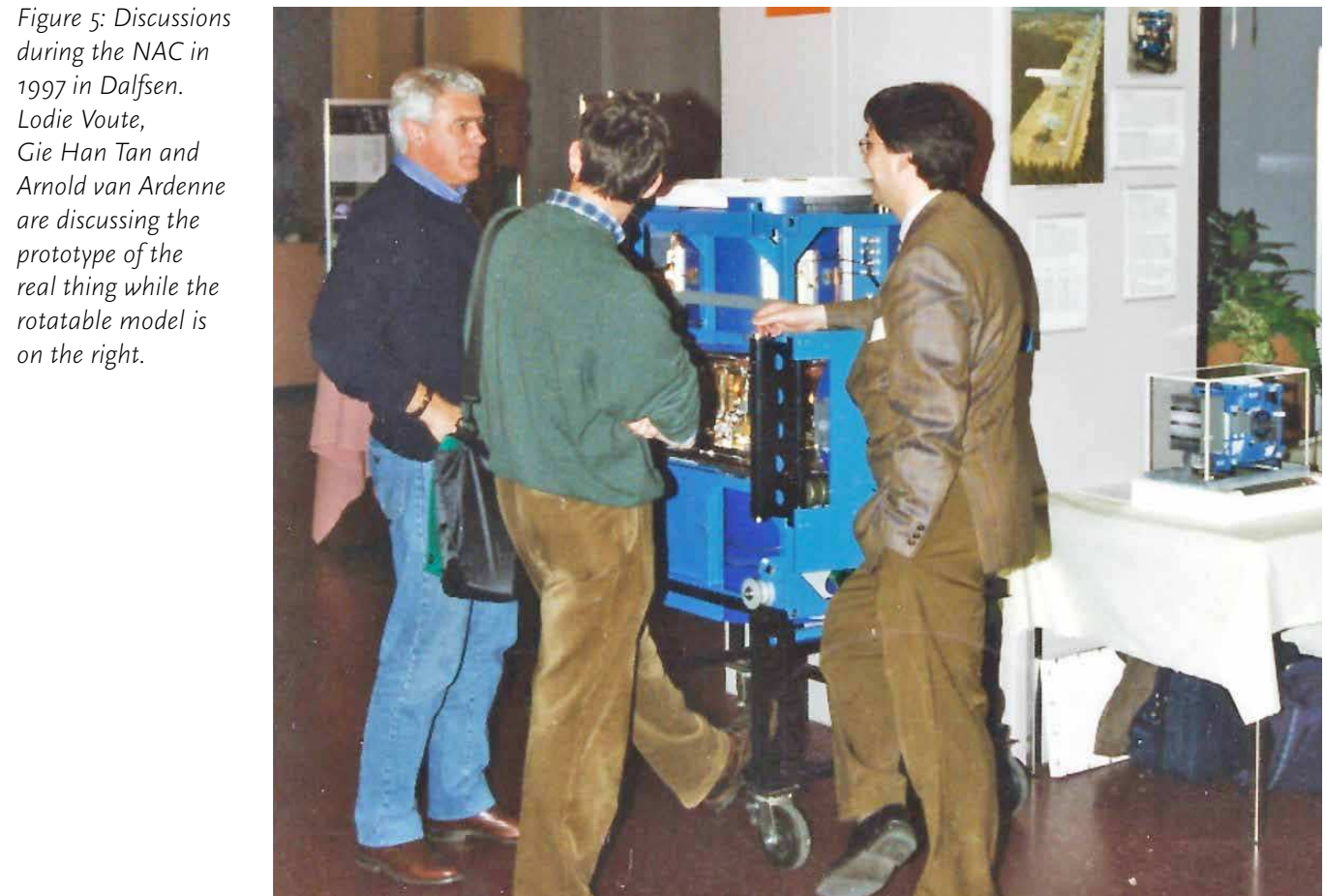

totype MFFE next to the rotatable model. On this occasion, the exhibition was on the first floor accessible by stairs and a small elevator. Going by the stairs was not an option so the prototype was fitted into the elevator by dismounting elements from both its sides?

In the end of the second phase, Gie Han, after a seconded stay at the German Company Rohde and Schwarz to develop the prototype LOFAR LBA antenna, left to join ESO. The last project phase was then capably lead by Yde Koopman and in 2001, the complete set of $12+2$ spare MFFE receivers were finished including their full cryogenic capability, and they were shipped to Westerbork. There, they were generally serviced under supervision of Harm-Jan Stiepel who mostly on his own, kept the WSRT receiver suites including the MFFE, alive and kicking. The picture (figure 6) shows Harm-Jan in the Westerbork electronics maintenance and support lab.

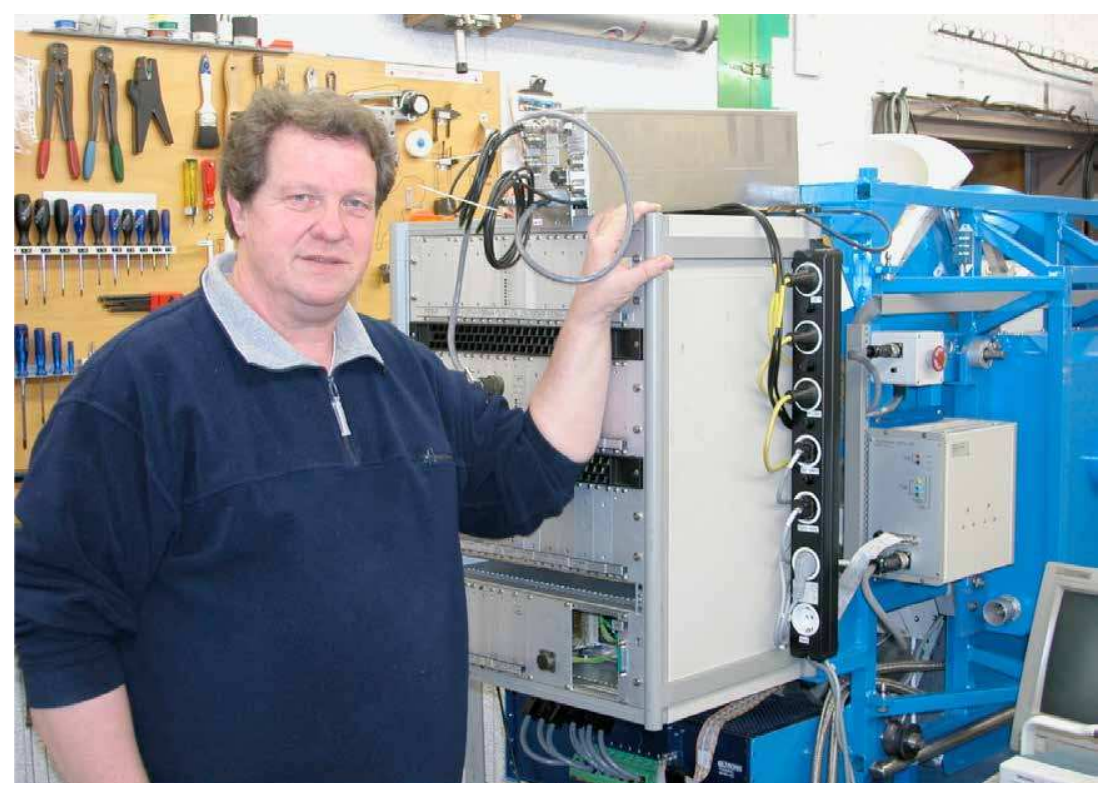

Figure 6: Harm Jan Stiepel in the Westerbork nance and suppor labs around 2005

It is interesting to note that Bert Woestenburg who was responsible for the many Low Noise Amplifiers and the cooled frontends throughout his career at ASTRON, identified about 70 ASTRON R\&D persons who contributed to the development of all WSRT receivers up to and including the MFFE. Over that period of close to 35 years, many people from other countries came to ASTRON's receiver lab to learn and collaborate, notably many from China's NAO for furthering Chinese radio astronomy.

While some of the MFFE still remain to be in use after almost 20 years, mostly for VLBI and geodetic observations, it can be said that in ASTRON the MFFE mark the end of an era and the start of the software receivers like Apertif and LOFAR. 


\section{The Life of the MFFE cryostat, from WSRT to ELT}

Temperature

Temperature for humans is often subjective and measured in relation to freezing water and boiling water. For instruments, temperature is -273.15 degree Celsius. Physical behaviour and technical performance are completely different at extreme low temperatures. For the WSRT, one crucial performance parameter affected by temperature is the noise of the system that should be as low as possible. Theoretically, the noise of receivers, antennas, filters and amplifiers is zero at zero Kelvin. Any higher temperature will produce noise. Building receivers, in particular low noise amplifiers, at hat minimum temperature has an mportant drawback. It would take huge amounts of energy and still the amplifier will not reach zero Kelvin.

In the total observing system the atmosphere above the WSRT has a temperature of, say, 213 to c). Fortunately, it is transparent to lower frequency radio waves so the around 10 Kelvin (depending on alt tude, wavelength, season, observing angle, rain, etc.). The reflector in the receiving system (the 25 meter absolute and starts at zero Kelvin or 300 Kelvin (about -60 to 27 degree effective "noise temperature" is only

diameter prime mirror) is also quite warm, from 273 to 300 Kelvin (o to 27 degree C), but given its good re flectivity the effective noise temperature is also around 10 Kelvin. The receiver noise temperature however should be even lower so that it contributes relatively little to the overal noise of the telescope system.

The Life of the WSRT testcryostat Our first experience at WSRT with cryogenically cooled front ends was during the 1980's and by the end of the 80's two telescopes were operating with helium cooling fridges and cold amplifiers. During the development of the Multi Frequency Front End (MFFE) for the WSRT in the 1990's, cryogenic systems were installed in all 14 telescopes. A test cryostat was designed and built to test receivers, LNA's, antennas, and filters intended for the MFFE. The box type cryostat had a large transparent entrance window from thin KAPTON foil supported by polystyrene foam, fully aluminium casing and as described in detail by Jaap Bregman in Internal Technical Report 215, it worked great. However, at the end it was still a bit too heavy and an update design based on honeycomb minium plates was implemented to

Figure 1: Box with MIRI grating wheel TVC

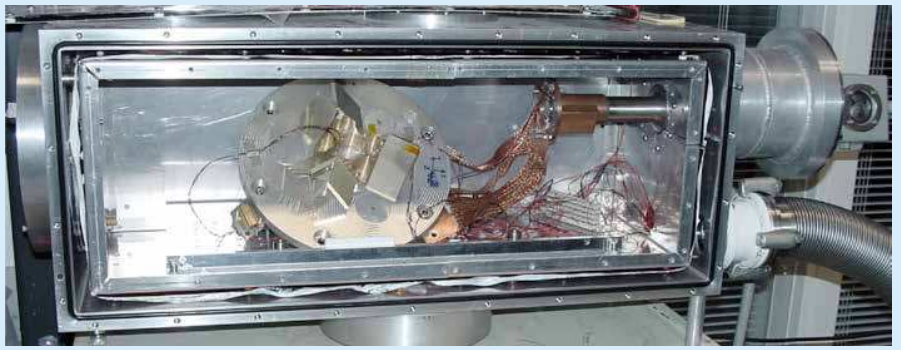
These new cryostats were implemented 16 times for all of the MFFE mented 16 times for all of the MFFE receivers (14 dishes, 2 spares). With MFFE up and running, the MFFE test cryostat went out of service.

\section{The Second Life:}

A second life for the test cryostat came soon after its service to the development of the MFFE. It would be used as a test system for an instrument destined for the Very Large Telescope (VLT) in Chile. The VLT Imager and Spectrometer for mid-Infrared (VISIR) was being developed at ASTRON. VISIR operates at mid Infrared wavelengths (humans feel this wavelength as cold (<40 Kelvin or -233 degrees C) so that the instrument structure and optics don't act like a sort of infrared lamp and over expose the detectors. The box cryostat entrance window was modified from the radio transparent thin Kapton foil to a thick flat transparent piece of Class (Fused Silica). It was then used to test aluminium mirrors, rotating long $(350 \mathrm{~mm}$ ) aluminium reflective grating (echelle) for the high resoluThe un of $200 \mathrm{~mm}$ and the deep structure of the cryostat box made it possib to install the echelle at its working angle. The box cryostat worked great! It was a relatively light weigh unit, easy to position in front of various optical test equipment such as the optical interferometer. The resolution of the aluminium optical unit could be tested while cooling. match the mass limits of the MFFE. warm radiation) and it needs to be mechanisms, and also the extreme
Even down to 40 Kelvin where we verified that it achieved the 30 nano meter resolution requirement.

\section{The Extended Life}

After the success of the VISIR tests, the test cryostat was modified again, this time to allow for a range of testing for other instruments. The MID-infrared Interferometric instrument (MIDI) is a two channel interferometer instrument for the VLT Interferometer (VLTI). We tested motors, special filters and grisms. For the VLT spectroscopic camera called SPIFFI $2 \mathrm{~K}$, the test cryostat was used to test shrinkage of plastic and glass down to 40 Kelvin while mounted in a very stiff cryogenic lens mount shown in Figure 2. This determined the design of a large glass lens $(185 \mathrm{~mm})$ in its aluminium housing. A test lens assembly was cooled and based on the postive results, plied to all five lenses of the $2 \mathrm{~K}$ camera [ref 1$]$ and worked great. It is still operational at the VLT! Th successful principal design was applied in several other cryogenic instruments to hold glass lenses and

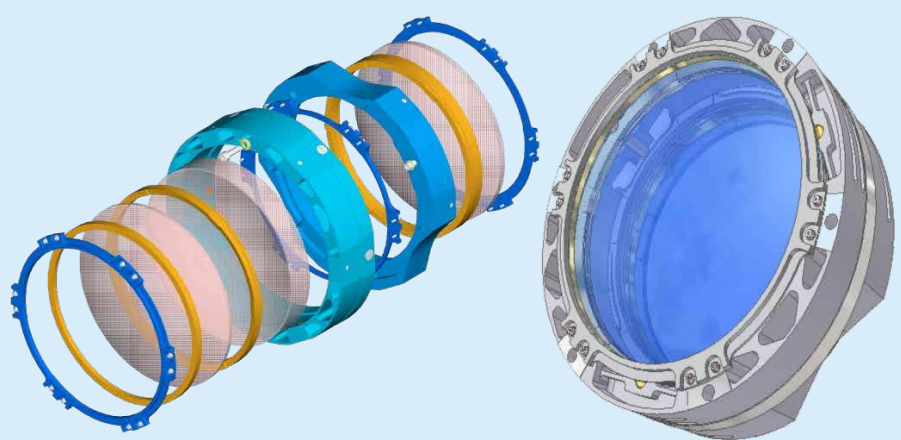

Figure 2: SPIFFI 2K Camera lensmount, typical size $185 \mathrm{~mm}$

prisms in aluminium structures. The cryostat is used for the space instrument JWST-MIRI (JWST is the successor of the Hubble Space telescope) to test optics. Ultimately for MIRI, a larger testcryostat is forsary to qualify the full instrument. This may look like the end for the box cryostat, but after 25 years of operation, it can still compete because of its easy handling and large entrance port.

The story continues with planned tests of the mirror for the METIS instrument and the MICADO ADC prototype both for the ELT and now in pre-design phase at NOVA-ASTRON.

This amazing test cryostat from our first experience with cryogenics on the WSRT in the 1980's has been used for testing 10 instruments over the years, and still counting!

ref 1: C. Kroes, et al., "Opto-Mechanical design for transmission optics in Cryo(n)
CHAPTER 10.2 THE MULTI FREQUENCY FRONTENDS 


\section{Acknowledgements}

Collaborations developed with all three Dutch Technical Universities for example regarding the triple band feed and the superconducting RFI filters. Some references are mentioned below but surely the MFFE could only be developed with many other colleagues not all mentioned here. The picture (Figure 7) shows the team of which most with some effort can be recognized. The commissioning phase had the involvement of many others from the Labs and Observatory staff.

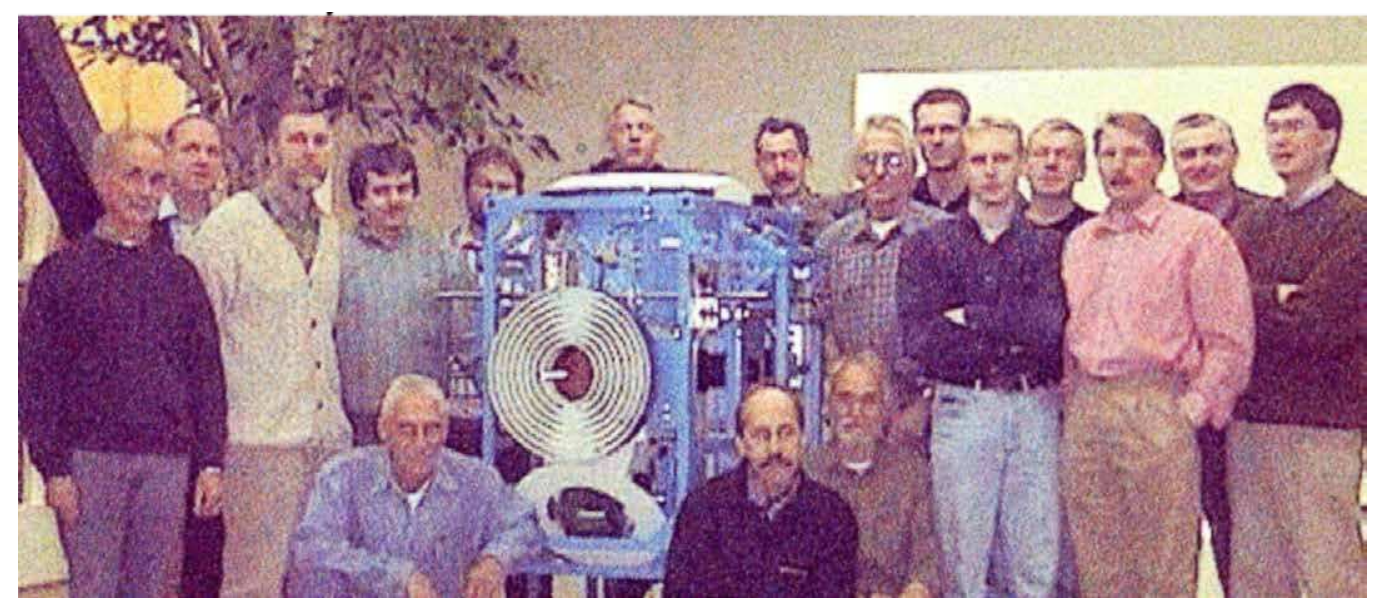

Figure 7: The MFFE team at the turn of the century in the last project phase, (left to right) Leo van der Ree, Yde Koopman, Paul Riemers, Jürgen Morawietz, Sieds Damstra (almost hidden), Anne Koster, Kees Brouwer, Martin Bakker, a student, Henk Heutink, Gie Han Tan and in the back row Menno Schuil, Lambert Nieuwenhuis and Ceorge Koenderink. Sitting in the front row: Jaap Bakker, Jan Idserda and Jan Dekker

Note (eds.): Cle Han Tan received the prestigious Dutch Veder Award in Jan. 2000 for his role in the MFFE development.

\section{References from Internal notes and reports:}

NFRA Notes

(http://www.astron.nl/r-d-laboratory/resultspublications/publications-pre-2009/notes/notes):

532 "Microstripsubstraten" (in Dutch) by A. Koeling

-500 "Brief outline of calibration system for new frontends" by A. van Ardenne

-572 "Noise source determination of $\mathrm{X}$ - and Y-dipole phase difference at $18 \mathrm{~cm}$ " by R.G. Strom

-640 "Calibratie voor de dubbele LNA-configuratie van de UHF-banden in het MFFE" (in Dutch) by

E.E.M. Woestenburg / A. Gunst

-521 "Metingen verricht aan een afstembare ontvanger voor de WSRT" (in Dutch) by G.H. Tan

-523 "Een nieuwe gecombineerd $18 \mathrm{~cm} / 21 \mathrm{~cm}$-feed" (in Dutch) by G.H. Tan

-618 "Beschrijving MFFE Controle Systemen Multi Frequency Frontend" (in Dutch) by A. Doorduin / R.

. R5. 597 "EMC/Lightning protection" resp. "EMC/System design MFFE" by G.H. Tan

-654 "Report of the UHF test period" by R.C. Strom
NFRA ITR's

(http://www.astron.nl//r-d-laboratory/resultspublications/publications-pre-2009/itrs/itrs ):

- 205 "A $230-460 \mathrm{MHz}$ HEMT- Amplifier with extremely Low Noise at Room Temperature" by E.E.M. Woestenburg

-206 "Cryogenic Noise Performance of HEMTs and MESFETs between 300 and 700 MHz" by E.E.M.

Woestenburg
-214 "Design Procedures for Long Endurance Cryostats with Large Microwave Windows." by J.D. Bregman

Other publications:

C.H. Tan, "Upgrade of the WSRT: The Multi Frequency Frontends", Proc. $26^{\text {th }}$ European Microwave Conference, pp59-64, Sept. 1996, Prague, Czech Rep.

C.H.Tan, "A Highly Stable, Phase Cohren, Wideband Microwave Synthesizer for Radio Astronomy Applications", Proc Joint EFTF-IEEE IFCS Meeting, pp 607-610, 1999

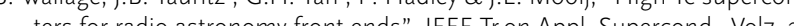




\section{Ever growing sensitivities}

\section{Chapter 10.3 WSRT surfing the digital wave}

André Gunst *

Figure 1: An early plot (end nineties) showing the nice fit of the capability trend of digital corLaw" forcurrent Law" for current and planned projects $S K A)$ $\perp$ oft

WSRT remained at the scientific foreground of discoveries by a process of enabling continuous improvements over its long history. One of the driving forces of these improvements was the understanding and application of the rapid advancements in digital technology. The digital electronics technology trend was nicely captured by the well-known "Moore's law" formulated already in 1965 by Intel founder Gordon Moore. It predicted the doubling of the amount of transistors within an integrated circuit every two years. Until recently, this "law" was followed by industrial developments. The WSRT digital instruments reaped all the benefits from that as shown in a plot by Arnold van Ardenne at the end-nineties in preparation for the SKA R\&D. The plot clearly shows a close connection between the ASTRON/JIVE and other digital correlators for radio astronomy and Moore's law. The graph also put plans for, at that time, future projects into perspective.

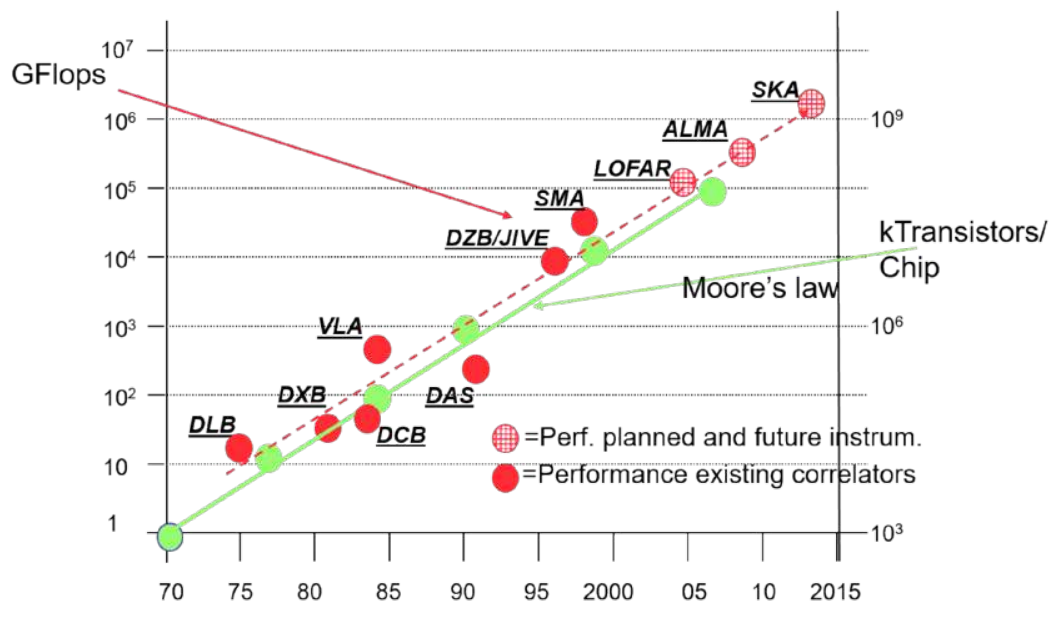

The digital electronic revolution started with Transistor-Transistor Logic (TTL) operating at 5 Volt. A the heart of this technology are transistors which are used as switches representing a "o" or a " 1 ". In the mid-seventies ASTRON's first digital correlator, the Digital Line Backend (DLB), used TTL circuitry with some functions integrated in an Application-Specific Integration Circuit (ASIC). The ASIC developed for the DLB remained on the commercial market for some years. In the early eighties, the follow-up technology was low power Schottky TTL logic (LS-TTL), characterized by up to three times higher switching rates. This LS-TTL was used extensively and successfully in the Digital Continuum Backend (DCB). As with the DCB, an ASTRON designed ASIC was used (see page 177 [Mr Correlator]).

The general need for a lower power solution gave birth to Complementary Metal Oxide Semiconductor (CMOS) technology, still used as workhorse technology today with increasingly smaller line definitions. In the DZB correlator, CMOS technology with a feature size of $800 \mathrm{~nm}$ is applied. The "Z" in the name DZB was chosen because it was the last correlator that Albert Bos (lead architect of all digital backends) designed for Westerbork. The CMOS technology in the DZB was integrated in a much more complex ASIC chip as well. The Haystack Observatory in Boston designed the overall ASICs, while using the correlator cell conceived by Albert Bos as a core. A total of 32 of these chips are integrated on boards and became operational in the nineties for both ASTRON, JIVE, MIT and the Submilimeter Array of the Smithsonian Institution in Hawaii.

As the computer industry required more processing per unit area, the transistor size in the CMOS technology was reduced. However, this led to increasing the nonrecurring costs for the masks which defined the pattern on the silicon chip, and hence the costs for the ASIC chips increased dramatically. For cost reasons it therefore was only attractive in cases where many chips were required. The industry solved this by the introduction of Field Programmable Gate Arrays (FPGA). An FPGA is an integrated circuit which is configurable after manufacture making it useful for many different applications.

Small FPGAs were already used in previous systems of the WSRT for auxiliary functions, but its first main use was in the Tied-Array Distribution Unit (TADU). In TADU, which became operational in 2006 , the functional density and performance was much increased by the use of the $130 \mathrm{~nm}$ FPGA from Altera.

The current Apertif system also uses FPGA with a CMOS technology of only $40 \mathrm{~nm}$. In total 8 FPGA's are integrated on a board: The UniBoard. Apertif is a very complex system with close to 1000 FPGA's in total.

The many generations of digital technology used in the WSRT inspired Sjouke Zwier to build a robot (see Figure 2). Actually, it was a robot statue, which is presently on display in front of the digital lab of ASTRON. 
Figure 2: On the right the robot statue built by Sjouk Zwier (on the left) together with Gils Schoonderbeek from many generations of left-over digital boards used in the AJDI Dec. 2016)

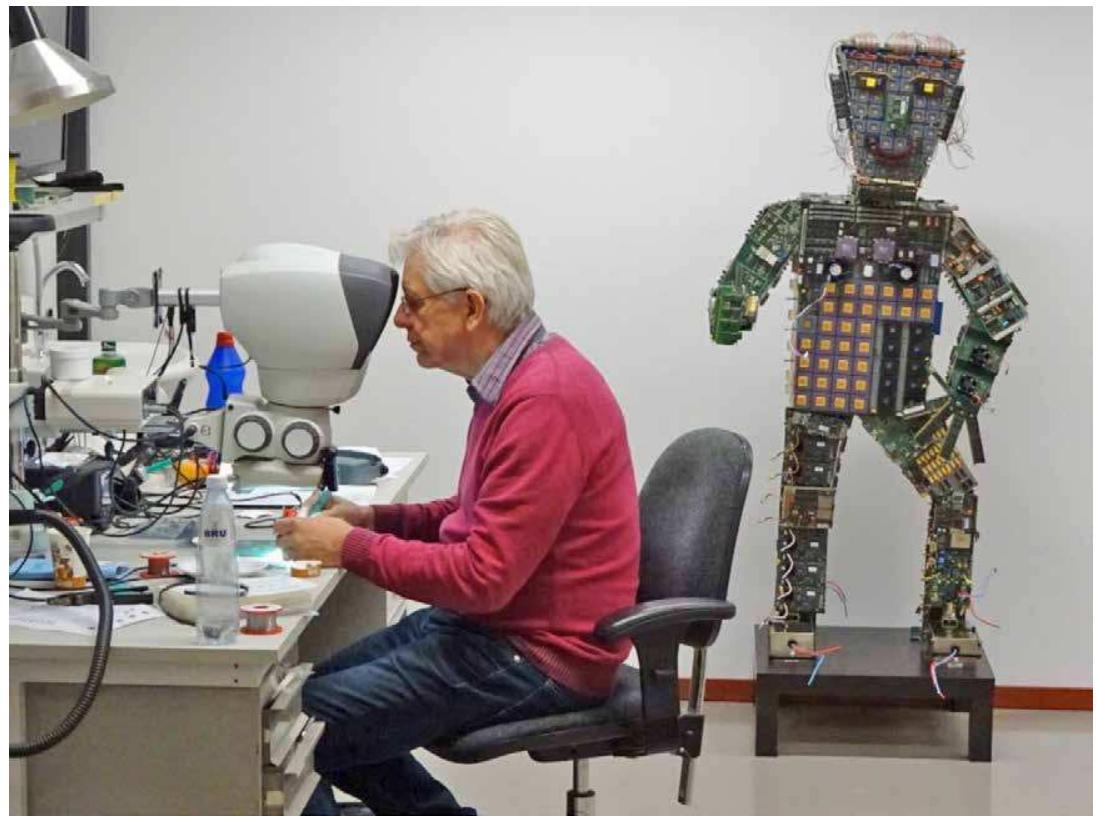

The robot was presented to the lab at the occasion of the retirement of Sjouke in December 2016.
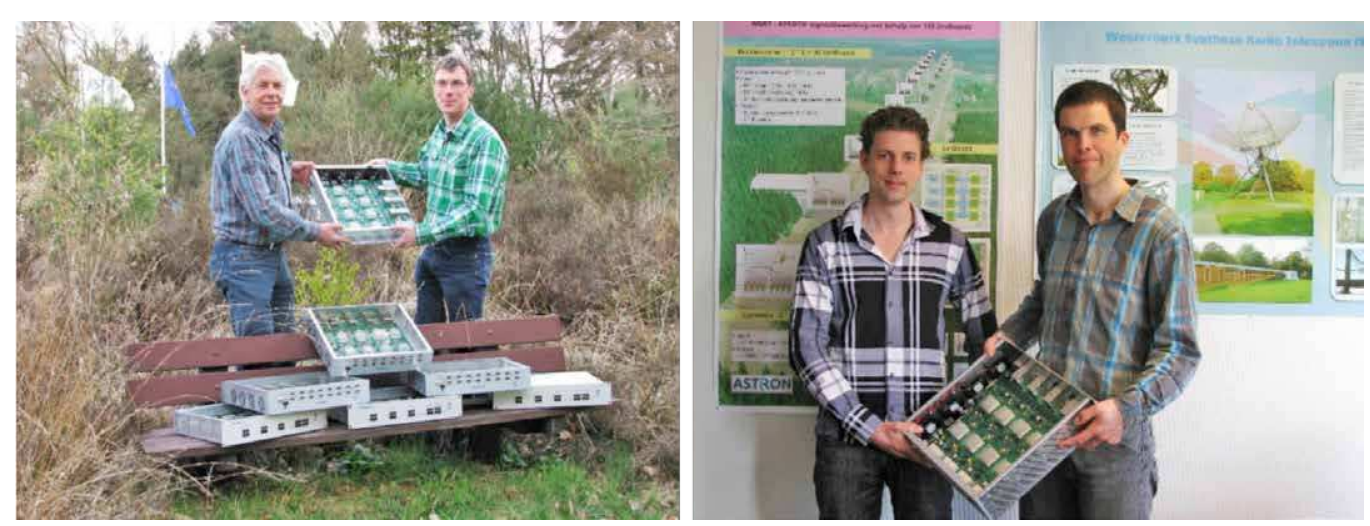

Figure 3: Various proud digital designers. On the left, the digital hardware designers Sjouke Zwier (I) and Ciijs Schoonderbeek (r). The right panel picture shows the digital firmware designers Daniël van der Schuur (I) and Eric Kooistra(r). They all are displaying the first UniBoard from different viewpoints.

The flexible FPGA processing Board (18 layers!) shown in Figure 3 reached an impressive $1 \mathrm{TBit} / \mathrm{sec}$ Input/output rate and consumes $200 \mathrm{~W}$. It is used in Apertif and for JIVE. A newer version with 3-4 times more processing power is used for ARTS (see Chapter 4). This successor, Uniboard 2 was developed in a 7partner collaboration in the Radionet EC-FP7 program.
The lessons learned from all these developments in their different applications, will be applied to the development of the SKA processing machines.
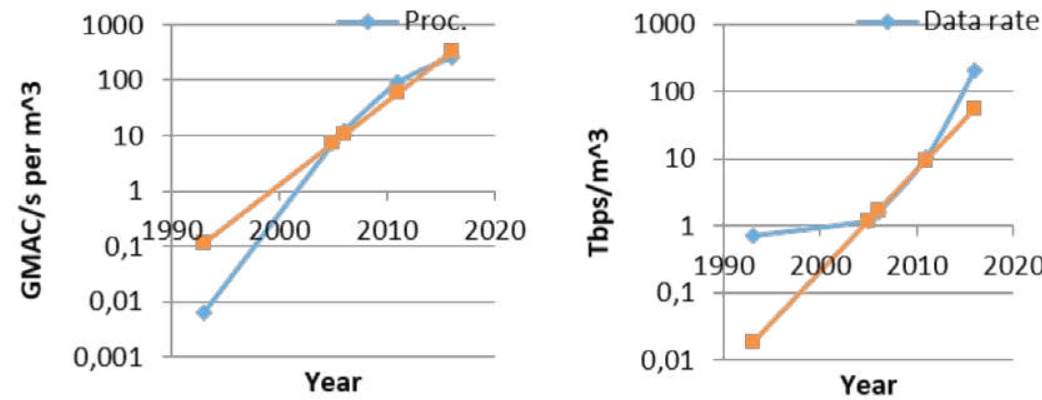

Figure 4: (left) Processing capability of hardware developed by RaDD (blue) and Moore's law (red) over the years, expressed in Giga Multiply and Accumulate per second and per m volume density. (right) Data rate capability of hardware developed by RaD (blue) and Moore's law (red) over the

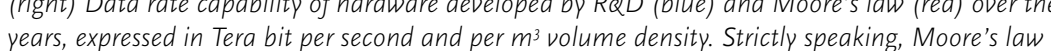
was meant for processing only.

As illustrated through Figure 4, many generations of digital electronics developed in-house have been used in the WSRT. This has been accomplished by applying the latest technology available by ASTRON and in collaborations. Hence, it is safe to say that this resulted successfully in surfing the digital waves, contributing to the radio astronomers quest to unveil the mysteries of the Universe.

As always it is not possible to acknowledge contributions of all of those who made this possible, but we acknowledge in particular Anne Koster, Arie Doorduin, Rob Millenaar, Albert Bos, André Kokkeler (now working at Twente University) and Hajee Pepping. 
